OKHEP-02-10

\title{
Does the Transverse Electric Zero Mode Contribute to the Casimir Effect for a Metal?
}

\author{
J. S. Høyє* \\ Department of Physics, Norwegian University of \\ Science and Technology, N-7491, Trondheim, Norway \\ I. Brevilit and J. B. Aarseth \\ Department of Energy and Process Engineering, \\ Norwegian University of Science and Technology, N-7491, Trondheim, Norway \\ K. A. Miltons \\ Department of Physics and Astronomy, \\ The University of Oklahoma, Norman, OK 73019 USA
}

(Dated: November 8, 2018)

\begin{abstract}
The finite temperature Casimir free energy, entropy, and internal energy are considered anew for a conventional parallel-plate configuration, in the light of current discussions in the literature. In the case of an "ideal" metal, characterized by a refractive index equal to infinity for all frequencies, we recover, via a somewhat unconventional method, conventional results for the temperature dependence, meaning that the zero-frequency transverse electric mode contributes the same as the transverse magnetic mode. For a real metal, however, approximately obeying the Drude dispersive model at low frequencies, we find that the zero-frequency transverse electric mode does not contribute at all. This would appear to lead to an observable temperature dependence and a violation of the third law of thermodynamics. It had been suggested that the source of the difficulty was the behaviour of the reflection coefficient for perpendicular polarization but we show that this is not the case. By introducing a simplified model for the Casimir interaction, consisting of two harmonic oscillators interacting via a third one, we illustrate the behavior of the transverse electric field. Numerical results are presented based on the refractive index for gold. A linear temperature correction to the Casimir force between parallel plates is indeed found which should be observable in room-temperature experiments, but this does not entail any thermodynamic inconsistency.
\end{abstract}

PACS numbers: 11.10.Wx, 05.30.-d, 73.61.At, 77.22.Ch

*Electronic address: johan.hoye@phys.ntnu.no

${ }^{\dagger}$ Electronic address: iver.h.brevik@mtf.ntnu.no

${ }^{\ddagger}$ Electronic address: jan.b.aarseth@mtf.ntnu.no

§Electronic address: milton@nhn.ou.edu 


\section{INTRODUCTION}

In spite of the numerous treatises on the Casimir effect during the past decade -for some books and review papers see, for instance, Milton [1], Mostepanenko and Trunov [2], Milonni [3], Plunien et al. [4], Bordag et al. [5] —it is somewhat surprising that such a basic issue as the temperature dependence of this effect is still unclear and has recently given rise to a lively discussion. This issue is not restricted to the case of curvilinear geometry, but is present even in the simplest conventional geometry of two parallel metal plates separated by a gap of width $a$. Thus Klimchitskaya and Mostepanenko in their detailed investigation [6], and also Bordag et al. 7], and Fischbach et al. [8], have argued that the Drude dispersion relation for a frequency-dispersive medium leads to inconsistencies in the sense that the reflection coefficient $r_{2}$ for perpendicular polarization (the TE mode) becomes discontinuous as the imaginary frequency $\zeta=-i \omega$ goes to zero. As is well known, the Drude dispersion relation reads for imaginary frequencies

$$
\varepsilon(i \zeta)=1+\frac{\omega_{p}^{2}}{\zeta(\zeta+\nu)},
$$

where $\omega_{p}$ is the plasma frequency and $\nu$ the relaxation frequency. (Usually, $\nu$ is taken to be a constant, equal to its room-temperature value.) The mentioned authors, instead of the Drude relation, give preference to the plasma dispersion relation, since no such discontinuity is then encountered. (In Ref. [9], the plasma relation together with the so-called surface impedance approach is argued to be the method best suited to describe the thermal Casimir force between real metals.) The plasma relation is

$$
\varepsilon(i \zeta)=1+\frac{\omega_{p}^{2}}{\zeta^{2}} .
$$

The arguments in Refs. [6, 7, 8, 9] are interesting, since they raise doubts not only about the applicability of the Drude model as such, but even more, doubt about the applicability of the fundamental Lifshitz formula at low temperatures (see, for instance, Ref. [10]).

The essence of the problem appears to be the following: For a metal, does the transverse electric (TE) mode contribute to the Casimir effect in the limit of zero frequency, corresponding to Matsubara integer $m=0$ ? It is precisely for this mode that the purported

discontinuity of the reflection coefficient $r_{2}$, mentioned above, can occur. The problem is most acute in the high $T$ regime (the $m=0$ contribution becomes increasingly important as $T$ increases), but is present at moderate and low temperatures as well. The conventional recipe for handling the two-limit problem for a metal, $n=\sqrt{\varepsilon} \rightarrow \infty, m \rightarrow 0$, has been to take the limits in the following order:

1. Set first $\varepsilon=\infty$;

2. then take the limit $m=0$.

This way of proceeding was advocated in the early paper of Schwinger, DeRaad, and Milton 11] (we will call it the SDM prescription), and was followed also in one of the recent papers by some of the current authors [12], and in Milton's recent book [1]. It seems to escaped recent notice that the physical basis for this prescription, namely the necessity of enforcing the correct electrostatic boundary conditions, was explicitly stated in Ref. [11]. 
Boström and Sernelius [13] seem to have been the first to inquire whether this prescription is right: They argued that in view of a realistic dispersion relation at low frequencies the $m=0$ TE mode should not contribute. And three of the present authors arrived recently at the same conclusion, in two papers dealing with the case of two concentric spherical surfaces 14, 15].

The Boström-Sernelius paper gave rise to a heated debate in the literature [7, 16, 17, 18] on the role of the $m=0 \mathrm{TE}$ mode for a metal. The advent of accurate experiments in recent years, by Lamoreaux [19], Mohideen et al. 20, 21, 22, 23], Ederth [24], Chan et al. [25], and Bressi et al. [26] (cf. also the recent review paper of Lambrecht and Reynaud [27]), represents important progress in this field. Especially the experiment of Bressi et al. is of interest in the present context, since it deals directly with the Casimir force between metal surfaces that are parallel, and so avoids use of complicating factors such as the proximity force theorem [28], which nevertheless seems well understood. This experiment is fraught with experimental difficulties (related to keeping the plates sufficiently parallel), so the accuracy is claimed by the authors to be moderate (15\%), but it is to be hoped that this accuracy will soon be improved. Several other related papers have appeared recently, discussing the interpretation of the mentioned experiments as well as more general aspects of finite temperature Casimir theory [29, 30, 31, 32, 33, 34, 35].

Our purpose in the present paper is to analyze the Casimir temperature problem anew, assuming conventional parallel-plate geometry from the outset, therewith avoiding the spherical Bessel functions that become necessary if spherical geometry is contemplated. In particular, we will focus attention on the $m=0$ TE mode. Let us summarize our results:

It is useful to distinguish between two different classes of metals. The first class, which we will call "ideal" metals, is characterized by a refractive index $n=\sqrt{\varepsilon}=\infty$ for all frequencies. It implies that the reflection coefficient $r_{2}$ mentioned above is unity for all $\zeta$. This corresponds to the traditional recipe 11 and 2 above when handling the two-limit problem for metals. It means that the $m=0 \mathrm{TE}$ mode contributes to the Casimir force just the same amount as does the transverse magnetic (TM) mode.

The obvious drawback of this "ideal" metal is that it does not occur in nature. And this brings us to the second class, which is the one of real metals, in which case we must observe an appropriate dispersion relation, especially at low frequencies. It is most commonly assumed that the most appropriate dispersion when $\zeta \rightarrow 0$ is the Drude relation, Eq. (1.1). As we will show, the Drude model implies that the $m=0 \mathrm{TE}$ mode does not contribute. The total $m=0$ free energy for a real metal becomes accordingly one half of the conventional expression. In contradistinction to recent statements in the literature [ 6 , 7, 8] we find that there exists no physical difficulty or ambiguity associated with the vanishing coefficient $r_{2}$ at $\zeta=0$. This is so because $r_{2}$ goes to zero smoothly when $\zeta \rightarrow 0$, as long as the transverse wave vector $\mathbf{k}_{\perp}$ is nonvanishing. (If $\mathbf{k}_{\perp}$ is precisely zero, there occurs a singularity in the reflection coefficient, but this has no physical importance since this point is of measure zero in the integral over $\mathbf{k}_{\perp}$.) Our present results are in agreement with Refs. 14, 15], as well as with Boström and Sernelius [13].

A different view has recently been put forward by Torgerson and Lamoreaux [36]. They argue that the Drude-model behavior does not accurately represent the TE zero mode, which necessarily has a vanishing tangential component at the surface of a perfect conductor. They point to the necessity of taking the finite thickness of the metallic coatings into account. Their arguments seem to imply that the conventional temperature dependence is correct. However, in our opinion electrostatic considerations of this kind do not solve the 
zero temperature problem; what is required to incorporate temperature dependence is an analytic continuation into imaginary frequencies of Green's functions referring to nonzero wavenumber.

Before embarking on the calculations let us emphasize the following point: The occurrence of the $m=0$ mode only once instead of twice is understandable physically. This mode is precisely the TM static mode, corresponding to the electric field being perpendicular to the two metal plates. It is the natural ground-state mode present when $\zeta=0$. Actually, in Sec. III of Ref. [12] we showed how the uniqueness of the static mode emerges naturally, using statistical mechanical considerations.

The outline of our paper is the following. In the next section we show why the exclusion of the TE zero mode seems to lead to an observable temperature correction to the force between real metal plates, and worse, seems to imply a violation of the third law of thermodynamics. In Sec. III we expand on the situation of an "ideal" metal in the sense described above, and calculate the Casimir free energy, entropy, and internal energy via a somewhat unconventional route. Equivalence with earlier results is demonstrated. In Sec. IV we introduce a new and simplified model to illustrate the Casimir problem, based essentially on statistical mechanics. In this model the system is replaced by two harmonic oscillators (the two media) that interact via a third oscillator (the electromagnetic field). Depending upon the form of the interaction we then have two situations. The first is the one where the induced interaction (or free energy), which is negative, increases linearly in magnitude with temperature in the classical limit. The other situation, which is more unexpected, is where the induced interaction vanishes in the classical limit. These two situations can be regarded as analogous to the behavior of the TM and TE modes. We also consider a strongly simplified case of real metals, and show how in such a case the contribution to entropy goes to zero smoothly as $T \rightarrow 0$. Arguing on basis of the Euler-Maclaurin formula we find this to be a general property (except in the idealized metal limit). We then go on to present numerical results based on the dispersion relation for gold, and obtain results qualitatively in accord with our analytical model. In the Appendices the smoothness of the reflection coefficient $r_{2}$, and of the TE Green's function, in the limit $\zeta \rightarrow 0$ is explicitly demonstrated. We also discuss the temperature dependence of the relaxation frequency, $\nu(T)$. We conclude that a linear temperature dependence should be observable in room temperature experiments.

In this paper we use natural units, $\hbar=c=k_{B}=1$.

\section{TEMPERATURE EFFECT FOR METAL PLATES}

We begin by reviewing how temperature effects are incorporated into the expression for the force between parallel dielectric (or conducting) plates separated by a distance $a$. To obtain the finite temperature Casimir force from the zero-temperature expression, one conventionally makes the following substitution in the imaginary frequency,

$$
\zeta \rightarrow \zeta_{m}=\frac{2 \pi m}{\beta}
$$

and replaces the integral over frequencies by a sum,

$$
\int_{-\infty}^{\infty} \frac{d \zeta}{2 \pi} \rightarrow \frac{1}{\beta} \sum_{m=-\infty}^{\infty}
$$


This reflects the requirement that thermal Green's functions be periodic in imaginary time with period $\beta$ [37]. Suppose we write the finite-temperature force/area as [for the explicit form, see Eq. (3.1) below]

$$
\mathcal{F}^{T}=\sum_{m=0}^{\infty} ' f_{m}
$$

where the prime on the summation sign means that the $m=0$ term is counted with half weight. To get the low temperature limit, one can use the Euler-Maclaurin (EM) sum formula,

$$
\sum_{k=0}^{\infty} f(k)=\int_{0}^{\infty} f(k) d k+\frac{1}{2} f(0)-\sum_{q=1}^{\infty} \frac{B_{2 q}}{(2 q) !} f^{(2 q-1)}(0),
$$

where $B_{n}$ is the $n$th Bernoulli number. This means here, with half-weight for the $m=0$ term,

$$
\mathcal{F}^{T}=\int_{0}^{\infty} f(m) d m-\frac{1}{2} f(0)+\frac{1}{2} f(0)-\sum_{k=1}^{\infty} \frac{B_{2 k}}{(2 k) !} f^{(2 k-1)}(0) .
$$

It is noteworthy that the terms involving $f(0)$ cancel in Eq. (2.4). The reason for this is that the EM formula equates an integral to its trapezoidal-rule approximation plus a series of corrections; thus the $1 / 2$ for $m=0$ in Eq. (2.2) is built in automatically. For a perfect conductor

$$
f(x)=-\frac{2}{\pi \beta} \int_{2 \pi x / \beta}^{\infty} q^{2} d q \frac{1}{e^{2 q a}-1} .
$$

Of course, the integral in Eq. (2.4) is just the inverse of the finite-temperature prescription (2.1b), and gives the zero-temperature result. The only nonzero odd derivative occurring is

$$
f^{\prime \prime \prime}(0)=-\frac{16 \pi^{2}}{\beta^{4}}
$$

which gives a Stefan's law type of term, seen in Eq. (2.10) below.

The problem is that the EM formula only applies if $f(m)$ is continuous. If we follow the argument of Ref. [13, 14, 15], and take the $\epsilon_{1,2} \rightarrow \infty$ limit at the end $\left(\epsilon_{1,2}\right.$ are the permittivities of the two parallel dielectric slabs), this is not the case, and for the TE mode

$$
\begin{aligned}
f_{0} & =0 \\
f_{m} & =-\frac{\zeta(3)}{4 \pi \beta a^{3}}, \quad 0<\frac{2 \pi a m}{\beta} \ll 1 .
\end{aligned}
$$

Then we have to modify the argument as follows:

$$
\begin{aligned}
\mathcal{F}^{T} & =\sum_{m=0}^{\infty}{ }^{\prime} f_{m}=\sum_{m=1}^{\infty} f_{m} \\
& =\sum_{m=0}^{\infty}{ }^{\prime} \tilde{f}_{m}-\frac{1}{2} \tilde{f}_{0},
\end{aligned}
$$

where $\tilde{f}_{m}$ is defined by continuity,

$$
\tilde{f}_{m}=\left\{\begin{array}{cc}
f_{m}, & m>0 \\
\lim _{m \rightarrow 0} f_{m}, & m=0
\end{array}\right.
$$


Then by using the EM formula,

$$
\begin{aligned}
\mathcal{F}^{T} & =\frac{\beta}{2 \pi} \int_{0}^{\infty} d \zeta f(\zeta)+\frac{\zeta(3)}{8 \pi \beta a^{3}}-\frac{\pi^{2}}{45}\left(\frac{a}{\beta}\right)^{4} \\
& =-\frac{\pi^{2}}{240 a^{4}}\left[1+\frac{16}{3}\left(\frac{a}{\beta}\right)^{4}\right]+\frac{\zeta(3)}{8 \pi a^{3}} T, \quad a T \ll 1
\end{aligned}
$$

The same result for the low-temperature limit is extracted through use of the Poisson sum formula, as, for example, discussed in Ref. [1]. Let us refer to these results, with the TE zero mode excluded, as the modified ideal metal model.

Exclusion of the TE zero mode will reduce the linear dependence at high temperature by a factor of two, but this is not observable by present experiments. The main problem, however, is that it adds a linear term at low temperature, which is given in Eq. (2.10), up to exponentially small corrections [1].

There are apparently two serious problems with the result (2.10):

- It would seem to be ruled out by experiment. The ratio of the linear term to the $T=0$ term is

$$
\Delta=\frac{30 \zeta(3)}{\pi^{3}} a T=1.16 a T
$$

or putting in the numbers $\left(300 \mathrm{~K}=(38.7)^{-1} \mathrm{eV}, \hbar c=197 \mathrm{MeV} \mathrm{fm}\right)$

$$
\Delta=0.15\left(\frac{T}{300 \mathrm{~K}}\right)\left(\frac{a}{1 \mu \mathrm{m}}\right),
$$

or as Klimchitskaya observed [38], there is a $15 \%$ effect at room temperature at a separation of one micron. One would have expected this to have been been seen by Lamoreaux [19]; his experiment was reported to be in agreement with the conventional theoretical prediction at the level of $5 \%$.

- Another serious problem is the apparent thermodynamic inconsistency. A linear term in the force implies a linear term in the free energy (per unit area),

$$
F=F_{0}+\frac{\zeta(3)}{16 \pi a^{2}} T, \quad a T \ll 1,
$$

which implies a nonzero contribution to the entropy/area at zero temperature:

$$
S=-\left(\frac{\partial F}{\partial T}\right)_{V}=-\frac{\zeta(3)}{16 \pi a^{2}} .
$$

Taken at face value, this statement appears to be incorrect. We will discuss this problem more closely in Sec. IV] and will find that although a linear temperature dependence will occur at room temperature, the entropy will go to zero as the temperature goes to zero. The point is that the free energy $F$ for a finite $\varepsilon$ always will have a zero slope at $T=0$, thus ensuring that $S=0$ at $T=0$. The apparent conflict with Eq. (2.13) or Eq. (2.10) is due to the fact that the curvature of $F(T)$ near $T=0$ becomes infinite when $\varepsilon \rightarrow \infty$. So Eqs. (2.12) and (2.13), corresponding to the modified ideal metal model, describe real metals approximately only for low, but not zero temperature-See, for example, Eq. (4.14). 


\section{CASIMIR FREE ENERGY, ENTROPY, AND INTERNAL ENERGY}

The Casimir surface force density $\mathcal{F}^{T}$ between two dielectric plates separated by a distance $a$ can be written as

$$
\mathcal{F}^{T}=-\frac{1}{\pi \beta} \sum_{m=0}^{\infty} \int_{\zeta_{m}}^{\infty} q^{2} d q\left[\frac{A_{m} e^{-2 q a}}{1-A_{m} e^{-2 q a}}+\frac{B_{m} e^{-2 q a}}{1-B_{m} e^{-2 q a}}\right] .
$$

(We follow the conventions of Ref. [39] and further references therein; here we further set $\hbar=c=1$.) The relation between $q$ and the transverse wave vector $\mathbf{k}_{\perp}$ is $q^{2}=k_{\perp}^{2}+\zeta_{m}^{2}$, where $\zeta_{m}=2 \pi m / \beta$. Furthermore

$$
\begin{aligned}
A_{m} & =\left(\frac{\varepsilon p-s}{\varepsilon p+s}\right)^{2}, \quad B_{m}=\left(\frac{s-p}{s+p}\right)^{2}, \\
s^{2} & =\varepsilon-1+p^{2}, \quad p=\frac{q}{\zeta_{m}},
\end{aligned}
$$

with $\varepsilon\left(i \zeta_{m}\right)$ being the permittivity. Note that whenever $\varepsilon$ is constant, the $A_{m}$ and $B_{m}$ depend on $m$ and $q$ only in the combination $p$,

$$
A_{m}(q)=A(p), \quad B_{m}(q)=B(p) .
$$

(This result may also be found in standard references such as Ref. [1].)

The free energy $F$ per unit area can be obtained from Eq. (3.1) by integration with respect to $a$ since $\mathcal{F}^{T}=-\partial F / \partial a$. We get 12 ]

$$
\beta F=\frac{1}{2 \pi} \sum_{m=0}^{\infty} \int_{\zeta_{m}}^{\infty}\left[\ln \left(1-\lambda^{\mathrm{TM}}\right)+\ln \left(1-\lambda^{\mathrm{TE}}\right)\right] q d q,
$$

where

$$
\lambda^{\mathrm{TM}}=A_{m} e^{-2 q a}, \quad \lambda^{\mathrm{TE}}=B_{m} e^{-2 q a} .
$$

(In the notation of Ref. [12], $\lambda_{\varepsilon} \equiv \lambda^{\mathrm{TM}}, \lambda \equiv \lambda^{\mathrm{TE}}$.)

From thermodynamics the entropy $S$ and internal energy $U$ (both per unit area) are related to $F$ by $F=U-T S$, implying

$$
S=-\frac{\partial F}{\partial T}, \quad \text { and thus } \quad U=\frac{\partial(\beta F)}{\partial \beta} .
$$

As mentioned above the behaviour of $S$ as $T \rightarrow 0$ has been disputed, especially for metals where $\varepsilon \rightarrow \infty$. We now see the mathematical root of the problem: The quantities $A_{m}=$ $B_{m} \rightarrow 1$ in the $\varepsilon \rightarrow \infty$ limit except that $B_{0}=0$ for any finite $\varepsilon$. So the question has been whether $B_{0}=0$ or $B_{0}=1$ or something in between should be used in this limit as results will differ for finite $T$, producing, as we saw above, a difference in the force linear in $T$. The corresponding difference in entropy will thus be nonzero. Such a difference would lead to a violation of the third law of thermodynamics, which states that the entropy of a system with a nondegenerate ground state should be zero at $T=0$. Inclusion of the interaction between the plates at different separations cannot change this general property. We will show that this discrepancy vanishes when the limit $\varepsilon \rightarrow \infty$ is considered carefully, by using the Euler-Maclaurin summation formula. Also, we will perform explicit analytic evaluation for any $T$ for metallic plates in the case where $\varepsilon \rightarrow \infty$ for all $\zeta$.

We will consider this latter case first. It is the case of "ideal" metals mentioned in Sec. [] and already considered briefly in Sec. II. 


\section{A. "Ideal" metals}

With $\varepsilon=\infty$ we have $A_{m}=B_{m}=1$ where we now also put $B_{0}=1$, i.e., $\lambda^{\mathrm{TM}}=\lambda^{\mathrm{TE}}=$ $e^{-2 q a}$. To remove the $\zeta$-dependence in the lower limit of integration in Eq. (3.4a), it is convenient to use the quantity $p$ of Eq. (3.2b) as a new variable. Expanding the logarithmic terms in Eq. (3.4a and keeping only the leading term, we get the task of calculating

$$
F \approx-\frac{1}{2 \pi \beta} I_{1}, \quad I_{1} \equiv 2 \sum_{m=0}^{\infty} \zeta_{m}^{2} \int_{1}^{\infty} p e^{-2 \gamma m p} d p
$$

where

$$
\gamma m=a \zeta_{m}=\frac{2 \pi a}{\beta} m
$$

Carrying out the integration in Eq. (3.6) we obtain

$$
I_{1}=\frac{1}{(2 a)^{2}} 2 \sum_{m=0}^{\infty}{ }^{\prime} L_{m}
$$

with

$$
L_{m}=(2 \gamma m+1) e^{-2 \gamma m} .
$$

(It is easy to check that this result is correct at $m=0$, where $p$ is not defined.) We encounter the following sums

$$
\begin{aligned}
& s_{0}(\gamma)=2 \sum_{m=0}^{\infty} e^{-2 \gamma m}=\operatorname{coth} \gamma \\
& s_{k}(\gamma)=2 \sum_{m=0}^{\infty}(2 \gamma m)^{k} e^{-2 m \gamma}=(-\gamma)^{k} \frac{\partial^{k} s_{0}}{\partial \gamma^{k}}
\end{aligned}
$$

so that

$$
\begin{aligned}
s_{1} & =\frac{\gamma}{\sinh ^{2} \gamma}, \\
s_{2} & =\frac{2 \gamma^{2} \cosh \gamma}{\sinh ^{3} \gamma}, \\
s_{3} & =\gamma^{3} \frac{6+4 \sinh ^{2} \gamma}{\sinh ^{4} \gamma} .
\end{aligned}
$$

The quantity $I_{1}$ is given by the first two of these sums,

$$
I_{1}(\gamma)=\frac{1}{(2 a)^{2}}\left[s_{1}(\gamma)+s_{0}(\gamma)\right]
$$

Alternatively, one could just first perform the summation in Eq. (3.6) (for $m \geq 1$ ) and then integrate. This summation yields $s_{2}(\gamma p)$. By subsequently integrating $s_{2}$ by parts the quantity $\left(s_{1}+s_{0}\right)$ in Eq. (3.11) is recovered (adding the $m=0$ term separately). 
By further expansion of the logarithm in Eq. (3.4a) one obtains terms $\lambda^{k} / k$ to be integrated and summed like Eq. (3.6). Performing the same steps as before, we find that the result (3.11) generalizes to

$$
F=-\frac{1}{8 \pi \beta a^{2}} \sum_{k=1}^{\infty} \frac{1}{k^{3}}\left[s_{1}(\gamma k)+s_{0}(\gamma k)\right],
$$

valid for arbitrary temperature.

The surface force per area (3.1) can now be obtained via $\mathcal{F}^{T}=-\partial F / \partial a$ utilizing $\gamma \propto a$ [Eq. (3.7)]. This yields

$$
\mathcal{F}^{T}=-\frac{1}{8 \pi \beta a^{3}} \sum_{k=1}^{\infty} \frac{1}{k^{3}}\left[s_{2}(\gamma k)+2 s_{1}(\gamma k)+2 s_{0}(\gamma k)\right] .
$$

The same result is also obtained by evaluating expression (3.1) (with $A_{m}=B_{m}=1$ ) in the same way as expression (3.4a) for $F$ was evaluated above. Using the second method, mentioned below Eq. (3.11), one finds that the integration of $s_{3}(\gamma p) / p$ yields the combination of $s_{i}$ present in Eq. (3.13).

Considering the $T \rightarrow 0$ limit, which implies the $\gamma \rightarrow 0$ limit, one obtains

$$
\mathcal{F}^{T}=-\frac{1}{8 \pi \beta a^{3}} \sum_{k=1}^{\infty} \frac{1}{k^{3}} \frac{6}{\gamma k}=-\frac{\pi^{2}}{240 a^{4}},
$$

using the limiting values of expressions (3.9a), (3.10a and (3.10b). This is the well known Casimir result for idealized metallic plates at $T=0$, seen in Eq. (2.10).

The internal energy $U$ is now found from Eqs. (3.5), (3.7), and (3.12) to be

$$
U=-\gamma^{2} \frac{\partial(F / \gamma)}{\partial \gamma}=-\frac{1}{8 \pi \beta a^{2}} \sum_{k=1}^{\infty} \frac{1}{k^{3}} s_{2}(\gamma k)
$$

and similarly an expression for the entropy $S$ follows from

$$
S=-2 \pi a \frac{\partial F}{\partial \gamma}=\frac{U-F}{T}=-\frac{1}{8 \pi a^{2}} \sum_{k=1}^{\infty} \frac{1}{k^{3}}\left[s_{2}(\gamma k)-s_{1}(\gamma k)-s_{0}(\gamma k)\right] .
$$

with Eqs. (3.12) and (3.15) inserted.

Now we can analyze the thermodynamic quantities in the low temperature limit using the properties of $s_{k}$ as defined by Eqs. (3.9a - (3.10c). We have for low temperature, ${ }^{1}$ where $\gamma \propto T \rightarrow 0$

$$
\begin{aligned}
& s_{0}=\frac{1}{\gamma}+\frac{1}{3} \gamma-\frac{1}{45} \gamma^{3}+\ldots, \\
& s_{1}=\frac{1}{\gamma}-\frac{1}{3} \gamma+\frac{1}{15} \gamma^{3}-\ldots, \\
& s_{2}=\frac{2}{\gamma}-\frac{2}{15} \gamma^{3}+\ldots, \\
& s_{3}=\frac{6}{\gamma}+\frac{2}{15} \gamma^{3}-\ldots
\end{aligned}
$$

\footnotetext{
${ }^{1}$ Actually, for a room-temperature experiment, $\gamma$ need not be small. For $T=300 \mathrm{~K}$ and $a=1 \mu \mathrm{m}$, $\gamma=0.823$.
} 
Inserting this into expressions (3.12), (3.13) or (3.15) one finds that the terms linear in $\gamma$ vanish. $^{2}$ Thus the entropy (3.16) vanishes, as it should in accordance with the third law of thermodynamics.

To obtain the leading correction to the $T=0$ result for finite $T$ one must consider the $\gamma^{3}$ term in the power series expansion of the summand in Eq. (3.13). However, the summation of this term with respect to $k$ diverges, ${ }^{3}$ because the expansion of $s_{n}(\gamma k)$ is not valid for large $k$. For small $\gamma$ one can instead integrate, without expanding, using the Euler-Maclaurin summation formula (2.3) to obtain a finite correction to the zero-temperature result. Using Eq. (2.3) to evaluate expression (3.13), the $\gamma \rightarrow 0$ expression (3.14) has to be subtracted to make $f(0)$ finite. Putting $x=\gamma k$ we have, apart from a prefactor,

$$
f(x)=\frac{1}{x^{3}}\left[s_{2}(x)+2 s_{1}(x)+2 s_{0}(x)-\frac{6}{x}\right],
$$

with $f(0)=-2 / 45$ in view of the expansions (3.17a - 3.17c). Integrating and using expressions (3.9a $)$, (3.10a $)$, (3.10b), we obtain

$$
\int_{0}^{\infty} f(x) d x=-\left.\frac{1}{x^{2}}\left[s_{1}(x)+s_{0}(x)-\frac{2}{x}\right]\right|_{0} ^{\infty}=0 .
$$

Including the $T=0$ result (3.14) we thus find

$$
\begin{aligned}
\mathcal{F}^{T} & =-\frac{1}{8 \pi \beta a^{3}}\left[\frac{6}{\gamma} \frac{\pi^{4}}{90}-\frac{1}{2} f(0) \gamma^{3}\right] \\
& =-\frac{\pi^{2}}{240 a^{4}}\left[1+\frac{1}{3}\left(\frac{2 a}{\beta}\right)^{4}\right], \quad a T \ll 1,
\end{aligned}
$$

where we have inserted expression (3.7) for $\gamma$ and noted that there is no $k=0$ term in Eq. (3.13), i.e., $f(0)$ is to be subtracted from expression (2.3). All the odd derivatives in the Euler-Maclaurin formula vanish because $f(x)$ is even. It should be noted that the expression for $\mathcal{F}^{T}$ is in agreement with what has been found earlier [cf. Eq. (2.10)], via alternative methods, by Milton [1], Klimchitskaya and Mostepanenko [6], Sauer [40], Mehra 41], and others, where the exponentially small correction to the above formula is also given.

The free energy (3.12) can be obtained from $\mathcal{F}^{T}=-\partial F / \partial a$, but this leaves a temperature dependent constant of integration. So instead we make use of the method above, where from Eq. (3.12)

$$
f(x)=\frac{1}{x^{3}}\left[s_{1}(x)+s_{0}(x)-\frac{2}{x}\right],
$$

and where now $f(0)=2 / 45$. With Eq. (3.21) we get a nonzero integral

$$
\begin{aligned}
C & =\int_{0}^{\infty} f(x) d x=-\int_{0}^{\infty} \frac{1}{x} \frac{d}{d x}\left(\frac{1}{x} \operatorname{coth} x-\frac{1}{3}-\frac{1}{x^{2}}\right) d x \\
& =\int_{0}^{\infty} \frac{1}{x^{3}}\left(\frac{1}{x}+\frac{x}{3}-\operatorname{coth} x\right) d x
\end{aligned}
$$

\footnotetext{
${ }^{2}$ This is actually stronger than necessary to insure vanishing entropy, since such terms would give $T^{2}$ terms in the energy or free energy.

${ }^{3}$ For this reason, the alternate expression (3.35) in Ref. 1] might be preferred. See Eq. (3.39) below.
} 
using partial integration. The integral (3.22) may be easily evaluated by contour methods. Due to symmetry the integral can be extended to minus infinity and then the contour of integration can be distorted into one which encircles the poles along the positive imaginary axis. Since $\operatorname{coth} z$ has poles at $z=i \pi m$ with $m$ integer we get ${ }^{4}$

$$
C=\frac{1}{2} 2 \pi i \sum_{m=1}^{\infty} \frac{-1}{(\pi i m)^{3}}=\frac{1}{\pi^{2}} \zeta(3)
$$

In view of this result as well as Eq. (3.14) we obtain for the free energy $(d k=d x / \gamma)$

$$
\begin{aligned}
F & =-\frac{1}{8 \pi \beta a^{2}}\left(\frac{2}{\gamma} \frac{\pi^{4}}{90}+\gamma^{3}\left(\frac{C}{\gamma}-\frac{1}{2} f(0)\right)\right) \\
& =-\frac{\pi^{2}}{720 a^{3}}\left(1+45\left(\frac{2 a}{\beta}\right)^{3} \frac{\zeta(3)}{\pi^{3}}-\left(\frac{2 a}{\beta}\right)^{4}\right), \quad a T \ll 1 .
\end{aligned}
$$

This result, including its exponentially small correction, is given in Ref. [1] and references therein. The internal energy $U$, which can be most easily be evaluated using Eq. (3.5), can also be computed by the method above, starting from the sum (3.15). Then

$$
f(x)=\frac{1}{x^{3}}\left(s_{2}(x)-\frac{2}{x}\right)=-\frac{1}{x^{2}} \frac{d}{d x}\left(s_{1}(x)+s_{0}(x)-\frac{2}{x}\right),
$$

with $f(0)=-2 / 15$. Partial integration replaces the $C$ of Eq. (3.22) with $-2 C$, and we obtain

$$
U=-\frac{\pi^{2}}{720 a^{3}}\left[1-90\left(\frac{2 a}{\beta}\right)^{3} \frac{\zeta(3)}{\pi^{3}}+3\left(\frac{2 a}{\beta}\right)^{4}\right], \quad a T \ll 1 .
$$

With Eq. (3.16) the entropy thus becomes (recall that $B_{0}=1$ is assumed)

$$
S=\frac{U-F}{T} \sim \frac{3 \zeta(3)}{2 \pi} T^{2}-\frac{4 \pi^{2} a}{45} T^{3}, \quad a T \ll 1 .
$$

\section{B. Equivalence with earlier results}

Equivalence with previous derivations can be shown for any $\gamma$. It is then convenient to utilize the Poisson summation formula. If $\tilde{c}(k)$ is the Fourier transform of $c(x)$, defined by

$$
\tilde{c}(k)=\int_{-\infty}^{\infty} d x c(x) e^{i k x}
$$

then

$$
\sum_{n=-\infty}^{\infty} c(n)=\sum_{m=-\infty}^{\infty} \tilde{c}(2 \pi m)
$$

\footnotetext{
${ }^{4}$ This low temperature $T^{3}$ dependence in $F$, which does not contribute to the force, is determined by the linear high temperature behavior of $\mathcal{F}^{T}$ - see Ref. [1], Sec. 3.2.1.
} 
With $c(x)=e^{-2 \gamma|x|}$ one finds

$$
\tilde{c}(2 \pi m)=\int_{-\infty}^{\infty} e^{-2 \gamma|x|+2 \pi m x i} d x=\frac{\gamma}{\gamma^{2}+(\pi m)^{2}}
$$

Thus

$$
\sum_{m=-\infty}^{\infty} \frac{\gamma}{\gamma^{2}+(\pi m)^{2}}=\sum_{n=-\infty}^{\infty} e^{-2 \gamma|n|}=\operatorname{coth} \gamma
$$

the familiar cotangent expansion, which can be verified in many different ways (cf. Ref. [42]).

In Eqs. (3.12) and (3.13) one of the sums is $\left[s_{0}(x)=\operatorname{coth} x\right]$

$$
S_{0}=\sum_{k=1}^{\infty} \frac{1}{k^{3}} \operatorname{coth}(\gamma k)=\sum_{k=1}^{\infty} \sum_{m=-\infty}^{\infty} S_{0 m k}
$$

where with Eq. (3.31)

$$
S_{0 m k}=\frac{\gamma k}{k^{3}\left[(\gamma k)^{2}+(\pi m)^{2}\right]}=\frac{1}{m u}\left[\frac{1}{k^{2}}-\frac{1}{k^{2}+(u / \pi)^{2}}\right], \quad u=\pi^{2} m / \gamma .
$$

Summation first with respect to $k$ where also the result (3.31) is utilized then gives

$$
S_{0 m}=\sum_{k=1}^{\infty} S_{0 m k}=\frac{1}{m u}\left[\frac{\pi^{2}}{6}-\frac{\pi^{2}}{2 u}\left(\operatorname{coth} u-\frac{1}{u}\right)\right] .
$$

In the limit $\gamma \rightarrow 0$ only the $m=0$ term remains, and we get the $T=0$ result if we use the expansion (3.17a) $(u \rightarrow 0)$

$$
S_{00} \rightarrow \frac{1}{m u}\left(-\frac{\pi^{2}}{2 u}\right)\left(-\frac{u^{3}}{45}\right)=\frac{\pi^{4}}{90} \frac{1}{\gamma}
$$

which is consistent with the $1 / k^{4}$ sum occurring in Eq. (3.14).

To obtain the free energy $F$ and the force $\mathcal{F}^{T}$ there are sums $S_{1}$ and $S_{2}$ that follow from the $s_{1}$ and $s_{2}$ of Eqs. (3.10a and (3.10b). And like Eqs. (3.32a) the relations between the various $s_{i}$ lead to

$$
S_{1 m}=-\gamma \frac{\partial}{\partial \gamma} g=u g^{\prime}
$$

where $g(u)=S_{0 m}$. Also:

$$
S_{2 m}=\gamma^{2} \frac{\partial}{\partial \gamma}\left(-\frac{u}{\gamma} g^{\prime}\right)=2 u g^{\prime}+u^{2} g^{\prime \prime}
$$

So to obtain $\mathcal{F}^{T}$ we need, because

$$
\left(2+4 u \frac{\partial}{\partial u}+u^{2} \frac{\partial^{2}}{\partial u^{2}}\right) \frac{1}{u^{2}} g(u)=g^{\prime \prime}(u),
$$

the combination

$$
\begin{aligned}
S_{2 m}+2 S_{1 m}+2 S_{0 m} & =\frac{\pi^{2}}{6 m} \frac{d^{2}}{d u^{2}}\left[u-3\left(\operatorname{coth} u-\frac{1}{u}\right)\right] \\
& =\frac{\pi^{2}}{m}\left(\frac{1}{u^{3}}-\frac{\cosh u}{\sinh ^{3} u}\right) \stackrel{m \rightarrow 0}{\longrightarrow} \frac{\pi^{2}}{m} \frac{u}{15}=\frac{\pi^{4}}{15} \frac{1}{\gamma}
\end{aligned}
$$


Altogether, restricting $m$ to positive values due to symmetry, the expression (3.13) can be reexpressed as $\left(u=\pi^{2} m / \gamma, \gamma=2 \pi a / \beta\right)$

$$
\mathcal{F}^{T}=-\frac{\pi^{2}}{240 a^{4}}\left[1+30 \sum_{m=1}^{\infty}\left(\frac{1}{u^{4}}-\frac{\cosh u}{u \sinh ^{3} u}\right)\right],
$$

which is the desired known expression. (For example, compare Eq. (3.35) of Ref. [1].)

To calculate the free energy (3.12) one likewise needs

$$
\begin{aligned}
S_{1 m}+S_{0 m} & =\frac{\pi^{2}}{6 m} \frac{d}{d u}\left[1-3\left(\frac{\operatorname{coth} u}{u}-\frac{1}{u^{2}}\right)\right] \\
& =\frac{\pi^{2}}{2 m}\left[\frac{\operatorname{coth} u}{u^{2}}+\frac{1}{u \sinh ^{2} u}-\frac{2}{u^{3}}\right] \\
& \stackrel{m \rightarrow 0}{\longrightarrow} \frac{\pi^{2}}{2 m}\left(-\frac{1}{45}+\frac{1}{15}\right) u=\frac{\pi^{4}}{45} \frac{1}{\gamma} .
\end{aligned}
$$

Thus the free energy becomes

$$
F=-\frac{\pi^{2}}{720 a^{3}}\left[1+45 \sum_{m=1}^{\infty}\left(\frac{\operatorname{coth} u}{u^{3}}+\frac{1}{u^{2} \sinh ^{2} u}-\frac{2}{u^{4}}\right)\right] .
$$

Compared with the small $T$ or $\gamma$ expansion (3.24) it is clear that the last term of Eq. (3.41) gives the $T^{4}=\beta^{-4}$ term of (3.24). The coefficient $C$ can also be identified from Eq. (3.41). As coth $u \rightarrow 1$ when $\gamma \rightarrow 0$ we must have, when comparing with Eq. (3.24),

$$
\left(\frac{\gamma}{\pi}\right)^{4} \frac{45 C}{\gamma}=45 \sum_{m=1}^{\infty} \frac{1}{u^{3}}=45\left(\frac{\gamma}{\pi^{2}}\right)^{3} \sum_{m=1}^{\infty} \frac{1}{m^{3}}
$$

or

$$
C=\frac{1}{\pi^{2}} \zeta(3)
$$

which is in agreement with Eq. (3.23).

\section{FINITE PERMITTIVITY. REAL METALS}

\section{A. Two harmonic oscillator models}

With finite permittivity $\varepsilon$ the $A_{m}$ and $B_{m}$ of Eq. (3.2a) will vary with $p$. Especially $B_{m} \rightarrow 0$ as $p \rightarrow \infty$ or $\zeta_{m} \rightarrow 0\left(\zeta_{m}=2 \pi m / \beta\right)$. In the high temperature or classical limit only the Matsubara frequency $\zeta=0$ (or $m=0$ ) can contribute as $\beta \rightarrow 0$. Thus, in the classical limit one has the result that the TE mode does not contribute at all. Physically, this means that the temperature becomes so high that only the static dipole-dipole interaction contributes (the $\zeta \rightarrow 0$ limit of the TM mode). In our opinion this somewhat unexpected behaviour is related to the peculiar type of interaction that exists between the canonical momentum $\mathbf{p}$ of a particle and the electromagnetic vector potential $\mathbf{A}(\mathbf{r}, t)$, which for a particle of mass $m$ and charge $q$ is $(\mathbf{p}-q \mathbf{A})^{2} / 2 m$. In addition to the standard cross term interaction $\mathbf{p} \cdot \mathbf{A}$ this also implies an interaction $\mathbf{A}^{2}$. 
As an illustration of the above we can consider two models, in each of which two harmonic oscillators interact via a third one. These oscillators represent a simplified picture of our polarizable parallel plates interacting via the electromagnetic field. The classical partition function of a harmonic oscillator with frequency $\omega$ is const $/(\beta \omega) \sim 1 / \sqrt{\omega^{2}}$, which gives a free energy $\sim \ln \left(\omega^{2}\right)$. Thus for three noninteracting harmonic oscillators the inverse partition function is proportional to $\sqrt{Q}$, where

$$
Q=a_{1} a_{2} a_{3}
$$

with

$$
a_{i}=\omega_{i}^{2}, \quad(i=1,2,3) .
$$

(The quantity $a_{3}$ corresponds to $k_{\perp}^{2}$ above.) By quantization using the path integral method 42, 43], the classical system is split into a set of harmonic oscillator systems described by Matsubara frequencies. Expression (4.1a) is replaced by

$$
Q=A_{1} A_{2} A_{3},
$$

where

$$
A_{i}=\omega_{i}^{2}+\zeta^{2}=a_{i}+\zeta^{2} .
$$

(For real frequencies, $\omega=i \zeta, 1 / A_{i}$ determines the response to an external oscillating force acting on the oscillator.)

Now add interactions, of strength proportional to $c$, between the third oscillator and the other two. The usual form of this interaction is $c x_{i} x_{j}$, where $x_{i}$ and $x_{j}$ are coordinates. Let this constitute the first model, which is analogous to the TM mode. Then the quantity $Q$ becomes the determinant of the matrix,

$$
\begin{aligned}
Q & =\left|\begin{array}{ccc}
A_{1} & 0 & c \\
0 & A_{2} & c \\
c & c & A_{3}
\end{array}\right|=A_{1} A_{2} A_{3}-c^{2}\left(A_{1}+A_{2}\right) \\
& =A_{1} A_{2} A_{3}\left(1-D_{1}\right)\left(1-D_{2}\right)\left(1-\frac{D_{1} D_{2}}{\left(1-D_{1}\right)\left(1-D_{2}\right)}\right)
\end{aligned}
$$

where

$$
D_{i}=\frac{1}{A_{i}} \frac{c^{2}}{A_{3}} \quad(i=1,2)
$$

The quantum free energy for this system of three coupled oscillators is given by summing over the Matsubara frequencies, as in Eq. (3.4a):

$$
\beta F=\frac{1}{2} \lim _{N \rightarrow \infty} \sum_{m=1}^{N}\left(\ln Q\left(\zeta_{m}\right)+3 \ln \eta^{2}\right),
$$

where $\eta=\beta / N$ and $\zeta^{2}$ is replaced by $2(1-\cos (\zeta \eta)) / \eta^{2}\left(=\zeta^{2}+\ldots\right)$ in the $A_{1} A_{2} A_{3}$ term of Eq. (4.3a ). The limiting procedure $N \rightarrow \infty$ is required to make the full free energy well defined. This means that the path integral representation of a harmonic oscillator is discretized by dividing the imaginary time of periodicity $\beta$ into $N$ pieces each of length $\eta$ as done in Ref. [42]. There, in an appendix an explicit evaluation was performed for one single oscillator. 
The various factors in Eq. 4.3a can be interpreted as follows: The product $A_{1} A_{2} A_{3}$ corresponds to the noninteracting system, the next two factors represent the result of interaction of single oscillators with the third one, while the last one is the contribution from the induced interaction between the two single oscillators via the third one. The logarithm of the last term is the analogue of the Casimir free energy. In this respect the term $c^{2} / A_{3}$ represents the induced interaction. Furthermore the $1 / a_{i}(i=1,2)$ represents the "bare" polarizability of noninteracting particles which for nonzero $\zeta$ becomes $1 / A_{i}$. Due to interaction with the "radiation" field this polarizability is modified into $1 /\left(A_{i}\left(1-D_{i}\right)\right)(i=1,2)$, where $D_{i}$ represents a "radiation" reaction from the "field" upon each single oscillator.

The above represents the ordinary situation, analogous to the TM mode. To model the TE mode, we can consider an analogy with the electromagnetic interaction in which the third oscillator can interact with the momenta of the first two. The analogous interaction will be $\left(p_{i} \text { - const. } x_{3}\right)^{2} / 2 m_{i} \quad\left(i=1,2 ; m_{i}\right.$ is mass $)$, including the unperturbed $p_{i}^{2}$ term. By evaluation of the classical partition function one now finds that the interaction from const. $x_{3}$ has no influence. (This is the analogue of classical diamagnetism which is equal to zero, as const. $x_{3}$ is seen to have no influence on the result when $p_{i}$ is integrated first.)

Quantum mechanically, the problem is a bit more complex. However, we can now exchange the roles of momenta and coordinates of the first two oscillators, i.e., we introduce a momentum representation. Then the interaction with the third oscillator can be written as $(i=1,2)$

$$
\text { const. } a_{i}\left(x_{i}-\frac{c}{a_{i}} x_{3}\right)^{2}=\text { const. }\left(a_{i} x_{i}^{2}-2 c x_{i} x_{3}+\frac{c^{2}}{a_{i}} x_{3}^{2}\right) \text {. }
$$

Now the last quadratic term adds to the energy of the third oscillator alone. Thus, compared to the first model considered above, $a_{3}$ is changed while the other $a_{i}$ remain unchanged:

$$
a_{3} \rightarrow a_{3}+c^{2} / a_{1}+c^{2} / a_{2} .
$$

Likewise in the quantum case

$$
A_{3} \rightarrow A_{3}+c^{2} / a_{1}+c^{2} / a_{2}
$$

The quantity $Q$ can still be written in the form (4.3a), but due to the change of $a_{3}$, the $\left(1 / A_{i}\right)(i=1,2)$ is replaced by $1 / A_{i}-1 / a_{i}=-\zeta^{2} /\left(a_{i} A_{i}\right)$ when evaluating $D_{i}$, i.e.,

$$
D_{i}=-\frac{\zeta^{2}}{a_{i}\left(a_{i}+\zeta^{2}\right)} \frac{c^{2}}{A_{3}} .
$$

The induced (analogous to the Casimir) free energy is again given by the logarithm of the third term in Eq. (4.3a). At zero and finite temperatures the latter logarithm is negative, and the free energy

$$
\frac{T}{2} \sum_{m=-\infty}^{\infty} \ln \left(1-\frac{D_{1} D_{2}}{\left(1-D_{1}\right)\left(1-D_{2}\right)}\right)
$$

is negative. Note that here the limiting procedure of Eq. (4.4) is not needed as sums for free energy differences converge, without difficulties. In the classical limit, however, the induced free energy becomes equal to zero $\left(D_{i} \rightarrow 0\right.$ implies that we get the logarithm of unity). We note the analogy: At high temperatures the same is true for the TE mode in the Casimir effect. There exists thus at least somewhere a finite temperature interval for which 
the Casimir free energy increases with increasing temperature. In turn, this means that the Casimir entropy $S=-\partial F / \partial T$ becomes negative in this interval.

This is a counterintuitive effect, but is physically due to the fact that we are dealing with the induced interaction part of the free energy of a composite system. We cannot apply usual thermodynamic restrictions such as positiveness of entropy to a "subsystem" of this sort. There exists actually a striking analogy with the peculiar formal properties one encounters in connection with the theory of the electromagnetic field in a continuous medium. The electromagnetic energy-momentum tensor that experimentally turns out to be definitely the best alternative when dealing with high-frequency effects, is the Minkowski tensor (cf., for instance, Ref. [44]). This tensor is however nonsymmetric, apparently breaking general conservation principles for angular momentum. The reason why this peculiar behaviour is yet quite legitimate physically, is that phenomenological electrodynamic theory is dealing only with a subsystem (the field itself plus its interaction with matter), and we cannot apply the same formal restrictions on it as we could if the system were closed.

\section{B. Real metal}

In the limit of an ideal metal $(\varepsilon \rightarrow \infty)$ the traditional (SDM) prescription, as mentioned in the Introduction, implies that $A_{m}=B_{m}=1$ for all $m$. In addition, as also mentioned previously, thermodynamic arguments have been given, claiming that the entropy does not become zero at $T=0$ in violation of the third law of thermodynamics if $B_{0}=0$ is used [35]. However, we do not find this to be the case; as we will show below, the entropy will be zero as required at $T=0$, even for a metal that is not idealized and where one bases the analysis on the value $B_{0}=0$.

Let us go back to Eq. (3.6). That equation was obtained by expanding Eq. (3.4a) to first order in $\lambda$ under the assumption that $A_{m}=B_{m}=1$. Doing the same expansion for finite permittivity, we obtain an integrand which contains a term with a factor $B_{m}$ (or $A_{m}$ ) that varies with $p=q / \zeta_{m}$ such that $B_{m} \rightarrow 0$ when $p \rightarrow \infty$. Expanding Eq. (3.4a) to higher order one obtains likewise powers of $B_{m}$ which, because $B_{m}<1$, become less important as compared to the case of an ideal metal (where $B_{m}=1$ ). One can first consider the case where $\varepsilon$ is independent of $\zeta$. When $\varepsilon$ is large one can use as a rough approximation

$$
B_{m}=\left\{\begin{array}{l}
1, p<\sqrt{\varepsilon} \\
0, p>\sqrt{\varepsilon}
\end{array}\right.
$$

This simple expression for $B_{m}$ is intended to show essential features that will be obtained

more accurately in a detailed numerical calculation. With this, Eq. (3.9a) (neglecting the influence of $A_{m}$ ) will turn into

$$
s_{0}(\gamma) \rightarrow s_{0}(\gamma)-s_{0}(\sqrt{\varepsilon} \gamma)=\operatorname{coth} \gamma-\operatorname{coth} \gamma_{c}
$$

with similar modifications for $s_{i}(i=1,2,3)$. Here

$$
\gamma_{c}=\gamma \sqrt{\varepsilon}
$$

is an effective sharp cutoff limit for the integral, a crude model for what should be a gradual cutoff for the integral of interest. [A gradual cutoff will only modify the last term of (4.10a) 
into a sum or integral over terms with varying $\gamma_{c}$. Namely, with varying $B=B(p)$, Eq. (3.6), if we recall the comment below Eq. (3.11), changes into $(B(1) \approx 1$ for $\varepsilon$ large)

$$
\begin{aligned}
I_{1} & =\frac{1}{(2 a)^{2}} \int_{1}^{\infty} B(p) s_{2}(\gamma p) \frac{d p}{p} \\
& =\frac{1}{(2 a)^{2}}\left[s_{0}(\gamma)+s_{1}(\gamma)\right]+\frac{1}{(2 a)^{2}} \int_{1}^{\infty}\left[s_{0}(\gamma p)+s_{1}(\gamma p)\right] B^{\prime}(p) d p,
\end{aligned}
$$

using partial integration. The approximation (4.9) means that $B^{\prime}(p)=-\delta(p-\sqrt{\varepsilon})$.]

As we did to obtain Eq. (3.27), we carry out the sum over $k$ in Eq. (3.16) while assuming $\varepsilon$ sufficiently large such that approximation (4.9) can be used. Then as in Eq. (4.10a) one obtains the previous result minus a term with $\gamma \rightarrow \gamma_{c}$. Keeping only the leading term, Eq. (3.27) is modified into

$$
S^{\mathrm{TE}} \sim \frac{3 \zeta(3)}{4 \pi}(1-\varepsilon) T^{2}, \quad \sqrt{\varepsilon} a T \ll 1 .
$$

[However, to be more accurate $B_{m}=((\sqrt{\varepsilon}-1) /(\sqrt{\varepsilon}+1))^{2}$ for $p=1$ and thus $B_{m}<1$ for $p<\sqrt{\varepsilon}$. When this is taken into account, we find that $S^{\mathrm{TE}} \propto-a \varepsilon^{5 / 2} T^{3}$ in a more narrow region, $\varepsilon^{3 / 2} a T \ll 1$, but that Eq. (4.12) holds for $\varepsilon^{-3 / 2} \ll a T \ll \varepsilon^{-1 / 2}$.]

Thus the entropy approaches zero as the temperature goes to zero. As $\varepsilon$ increases the $T$ dependence becomes more singular, because the region in which Eq. (4.12) is valid becomes more and more narrow, but the value at $T=0$ stays fixed at zero also in the limit $\varepsilon \rightarrow \infty$. This contrasts the ideal metal result (2.13) where $\varepsilon=\infty$ is used.

Again, we note the counterintuitive negative contribution from the TE mode. As mentioned earlier, this does not violate the laws of thermodynamics and can be understood in terms of the oscillator model analysed in some detail in Sec. IVA. Only the total entropy has to increase with increasing temperature. And this is the case for the inverse partition function (4.3a) which represents three interacting harmonic oscillators where the $D_{i}$ are given by Eq. (4.7). Although the induced entropy becomes negative at least in some finite temperature region the total entropy will behave properly, as the total system can be decomposed into three independent harmonic oscillators represented by the eigenvalues of the matrix (4.3a ) with $A_{i}$ replaced by $a_{i}(i=1,2)$, and furthermore $A_{3}$ replaced by the right hand side of Eq. (4.6a).

With the simplification (4.9) for the TE-mode the free energy can be easily expressed in terms of the "ideal" metal case analysed in Sec. IIIA Let the "ideal" metal free energy be $F=F_{I}(T)$. From Eq. (3.7) $\gamma \propto T$. Now the magnification of $\gamma$ to $\gamma_{c}$ as in Eq. (4.10b) and insertion of it in Eq. (3.12) will change the corresponding free energy to $\left(\gamma / \gamma_{c}\right) F_{I}\left(T \gamma_{c} / \gamma\right)=$ $F_{I}(\sqrt{\varepsilon} T) / \sqrt{\varepsilon}$. The TM- and TE-modes both contribute the same amounts to (3.12). Thus with Eq. (4.9) the free energy will be

$$
F=F(T)=F_{I}(T)-\frac{1}{2 \sqrt{\varepsilon}} F_{I}(\sqrt{\varepsilon} T)
$$

From this we have (keeping in each case only the leading temperature correction)

$$
F(T)= \begin{cases}(1-1 /(2 \sqrt{\varepsilon})) F_{I}(0)-\frac{\zeta(3)}{4 \pi}(2-\varepsilon) T^{3}, & 0 \leq a T \ll 1 / \sqrt{\varepsilon}, \\ F_{I}(0)+K_{I} T / 2, & 1 / \sqrt{\varepsilon} \ll a T \ll 1, \\ -K_{I} T / 2, & 1 \ll a T .\end{cases}
$$


where the constant $K_{I}=\zeta(3) /\left(8 \pi a^{2}\right)$ is the magnitude of the slope of the linear dependence of the high temperature result of the the "ideal" metal $\left(F_{I}(0)=-\frac{\pi^{2}}{720 a^{3}}<0\right)$. Thus for high temperatures non-ideal or realistic metals yield one half of the "ideal" metal result. The intermediate form, which holds at room temperature, is the same as seen in Eq. (2.12). Again, we see that in the $\sqrt{\varepsilon} a T \ll 1$ regime the result (4.12) for the entropy holds. [Equation (4.14) includes the TM mode as well.]

Now, $\varepsilon$ usually depends on $\zeta$. But this will not change our conclusions from Eq. (4.12). To see this we can go back to expression (3.6) which followed from expansion of the logarithmic term in the free energy (3.4a). In the general case, the coefficients $A_{m}$ and $B_{m}$, which are less than 1, should be included in Eq. (3.6) , and powers of them will occur in the evaluation of the terms contributing to the free energy for $k>1$. These factors will all be smooth functions of $\zeta$ except for the case of an idealized metal where $B_{m}$ becomes discontinuous at $\zeta=0$. This smoothness is also valid for the Drude formula discussed in Appendix $\mathrm{A}$. With $A_{m}$ and $B_{m}$ included, Eq. (3.4a) can be summed with respect to $\zeta_{m}=2 \pi m / \beta$, and the Euler-Maclaurin formula (2.3) can again be applied. (Equation (3.6) with $B_{m}$ included is not applicable in this situation as we remarked there because $\zeta \rightarrow 0$ is of relevance here.) If $\varepsilon$ stays finite when $\zeta \rightarrow 0$ the result clearly will be the same as that given above. However, for a real metal where $\varepsilon \rightarrow \infty$ as $\zeta \rightarrow 0$ the situation is more subtle. For the case of an ideal metal considered in Sec. III, the first derivative $f^{\prime}(0)$ was zero while $f^{\prime \prime \prime}(0)$ of Eq. (2.6) was nonzero. By similar application of the Euler-Maclaurin formula to the free energy (3.4a) instead of the force (3.1), the same will be true. For a real metal obeying the Drude dispersion relation (1.1) (with $\nu \neq 0$ ) the first derivative $f^{\prime}(0)$ continues to be zero due to the $\zeta$ dependence of $B_{m}, B_{m} \sim \zeta_{m}^{2}$, according to Eq. A4 . Thus, quite generally, we expect a $T^{3}$ (or $T^{4}$ ) correction to the free energy at sufficiently low temperature.

\section{Gold as a numerical example}

Let us go back to Eq. (3.1) for the surface force density, making use of the best available experimental results for $\varepsilon(i \zeta)$ as input when calculating the coefficients $A_{m}$ and $B_{m}$. We choose gold as an example. Useful information about the real and imaginary parts, $n^{\prime}$ and $n^{\prime \prime}$, of the complex permittivity $n=n^{\prime}+i n^{\prime \prime}$, versus the real frequency $\omega$, is given in Palik's book [45] and similar sources. The range of photon energies given in Ref. [45] is from 0.1 $\mathrm{eV}$ to $10^{4} \mathrm{eV}$. (The conversion factor

$$
1 \mathrm{eV}=1.519 \times 10^{15} \mathrm{rad} / \mathrm{s}
$$

is useful to have in mind.) When $n^{\prime}$ and $n^{\prime \prime}$ are known the permittivity $\varepsilon(i \zeta)$ along the positive imaginary frequency axis, which is a real quantity, can be calculated by means of the Kramers-Kronig relations.

Figure 1 shows how $\varepsilon(i \zeta)$ varies with $\zeta$ over seven decades, $\zeta \in\left[10^{11}, 10^{18}\right] \mathrm{rad} / \mathrm{s}$. The curve was given in an earlier paper [30], and is reproduced here for convenience. (We are grateful to A. Lambrecht and S. Reynaud for having given us the results of their accurate calculations.) At low photon energies, below about $1 \mathrm{eV}$, the data are well described by the Drude model, Eq. (1.1), in which the input parameters have the values 30]

$$
\omega_{p}=9.0 \mathrm{eV}, \quad \nu=35 \mathrm{meV} .
$$

These values refer to room temperature. The curve in Fig. 1 shows a monotonic decrease of $\varepsilon(i \zeta)$ with increasing $\zeta$, as any permittivity along the positive imaginary axis has to follow 


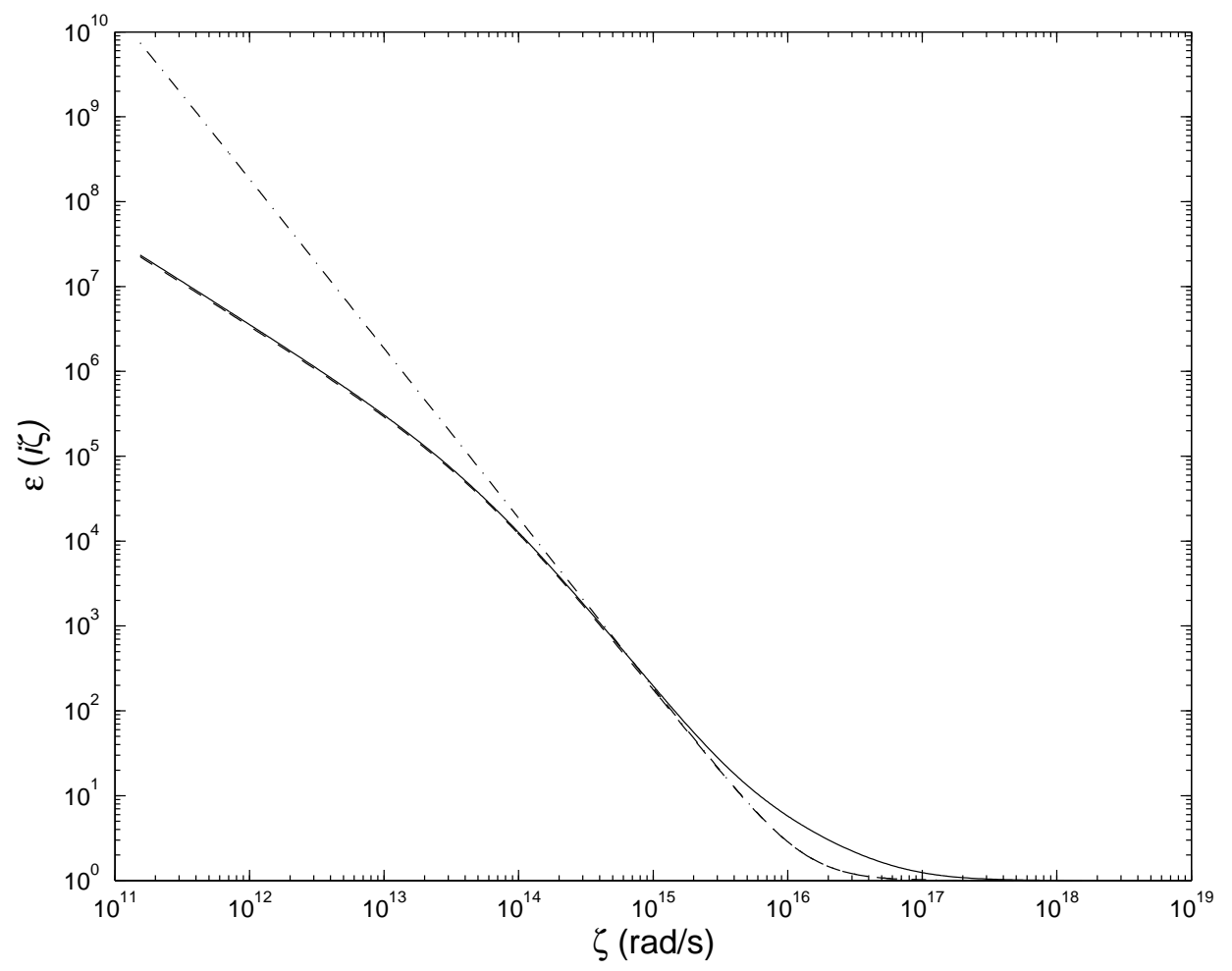

FIG. 1: Full line: Permittivity $\varepsilon(i \zeta)$ as function of imaginary frequency $\zeta$ for gold. The curve is calculated on the basis of experimental data. Courtesy of Astrid Lambrecht and Serge Reynaud. Broken lines: $\varepsilon(i \zeta)$ versus $\zeta$ with $T$ as parameter, based upon the temperature dependent Drude model; cf. Appendix D The upper curve is for $T=10 \mathrm{~K}$; the lower is for $T=300 \mathrm{~K}$, which for energies below $1 \mathrm{eV}\left(1.5 \times 10^{15} \mathrm{rad} / \mathrm{s}\right)$ nicely fits the experimental data. Both curves are below the experimental one for $\zeta>2 \times 10^{15} \mathrm{rad} / \mathrm{s}$.

according to thermodynamical requirements. The two broken curves in the figure show, for comparison, how $\varepsilon(i \zeta, T)$ varies with frequency if we accept the Drude model for all frequencies, and include the temperature dependence of the relaxation frequency with $T$ as a parameter. Cf. Appendix D. For $T=300 \mathrm{~K}$, the Drude curve is seen to be good for all frequencies up to $\zeta \sim 2 \times 10^{15} \mathrm{rad} / \mathrm{s}$; for higher $\zeta$ it gives too low values of $\varepsilon$. Both Drude curves, for $T=10 \mathrm{~K}$ and $T=300 \mathrm{~K}$, are seen to give the same values when $\zeta \geq 3 \times 10^{14}$ $\mathrm{rad} / \mathrm{s}$.

The structure of Eq. (3.1) shows that for numerical integration it is advantageous to introduce the nondimensional quantity

$$
y=q a
$$

as the integration variable. The force expression then takes the form

$$
\mathcal{F}^{T}=-\frac{1}{\pi \beta a^{3}} \sum_{m=0}^{\infty} \int_{m \gamma}^{\infty} y^{2} d y\left[\frac{A_{m} e^{-2 y}}{1-A_{m} e^{-2 y}}+\frac{B_{m} e^{-2 y}}{1-B_{m} e^{-2 y}}\right] .
$$

(This formula holds even when practical units are restored, when $\beta=1 / k_{B} T$.) Typical magnitudes of the attractive pressure are about one millipascal, for a gap width of $1 \mu \mathrm{m}$. (The force between ideal metal plates at zero temperature for $1 \mu \mathrm{m}$ separation is $1.30 \mathrm{mPa}$.) 
The next task is to determine the values of $A_{m}$ and $B_{m}$, in the limiting case of $m \rightarrow 0$. This has to be done analytically. Whereas the TM mode leads unambiguously to $A_{0}=1$ $(\varepsilon \gg 1)$, the TE mode is more delicate. In Sec. A we show explicitly, by means of a limiting procedure based on the Drude model, how $B_{m} \rightarrow 0$ when $\zeta \rightarrow 0$, i.e., when $m \rightarrow 0$. The $m=0$ TE mode accordingly does not contribute. To summarize:

$$
\begin{aligned}
& A_{0}=1, \quad B_{0}=0 \quad \text { for a metal } \quad(\varepsilon(0)=\infty) \\
& A_{0}=\left(\frac{\varepsilon-1}{\varepsilon+1}\right)^{2}, \quad B_{0}=0 \text { for a dielectric medium }(\varepsilon=\varepsilon(0)) .
\end{aligned}
$$

These relations will be assumed in the following.

There are some general properties of the expression (4.18) that ought to be noticed. First, at the lower limit, $y=m \gamma$, the coefficients $A_{m}$ and $B_{m}$ for $m \geq 1$ become equal,

$$
A_{m}=B_{m}=\left(\frac{\sqrt{\varepsilon}-1}{\sqrt{\varepsilon}+1}\right)^{2}, \quad \varepsilon=\varepsilon\left(i \zeta_{m}\right) .
$$

This expression is precisely the reflection coefficient for Poynting's vector, at normal incidence. This special case obviously corresponds to $\mathbf{k}_{\perp}=\mathbf{0}$. Then the TE and TM modes are identical to each other. Secondly, we note that for large values of $y$, the integrand in Eq. (4.18) approaches

$$
\left(\frac{\varepsilon-1}{\varepsilon+1} \frac{y}{e^{y}}\right)^{2}, \quad \varepsilon=\varepsilon\left(i \zeta_{m}\right),
$$

showing how quickly the contributions from large $y$ die out.

The full line in Fig. 2 shows how the magnitude of $\mathcal{F}^{T}$ for gold varies with the dimensionless parameter $a T$, when $a=1 \mu \mathrm{m}$. The lower limit $a T=4.4 \times 10^{-3}$ corresponds to the low temperature of $T=10 \mathrm{~K}$. Terminating the $y$ integration at the upper limit $y_{\max }=30$ we found the necessary number of terms in the $m$ sum to be about $N=450$. At room temperature, $T=300 \mathrm{~K}$, corresponding to $a T=0.131$ and $\gamma=0.823$, the required number of terms was found to be lower, $N=15$ (assuming the same $y_{\max }$ ). In the upper limit, $a T=0.52(T=1200 \mathrm{~K})$, only $N=4$ was required. This property of only a small number of terms being necessary at high temperatures is as we would expect. Note, however, that the temperature variation of $\varepsilon(i \zeta)$ is not taken into account. The only known empirical data for $\varepsilon(i \zeta)$ are referring to room temperature, and are as given in Fig. 1,

The broken line in the same figure gives the result calculated from the expression in Eq. (2.10), which is for the modified ideal metal model in which the TE zero mode has been removed. The deviations from the full lines are seen to be quite uniform: $13 \%$ at the lower limit, $12 \%$ at room temperature, and $18 \%$ at the upper limit. This uniformity in the deviations is somewhat surprising, in view of the fact that the expression (2.10) is a low-temperature expansion which one would expect to be most accurate when $a T \rightarrow 0$. The reason for the deviations must lie in the different ways the two force expressions are calculated: Eq. (2.10) is based upon the idealized assumptions $A_{m}=B_{m}=1$ for all $\mathrm{m}$ except that $B_{0}=0$, whereas Eq. (4.18) is calculated using the realistic dispersive data from Fig. 1, plus Eq. (4.19a) in the case $m=0$.

Figure 3 shows that the behaviour is essentially the same if the gap is made wider, $a=4 \mu \mathrm{m}$. The forces are now only about $0.4 \%$ of those in Fig. 2. The lower limit $a T=0.017$ corresponds to $T=10 \mathrm{~K}(N=115$ terms necessary), and the upper limit $a T=0.523$ 


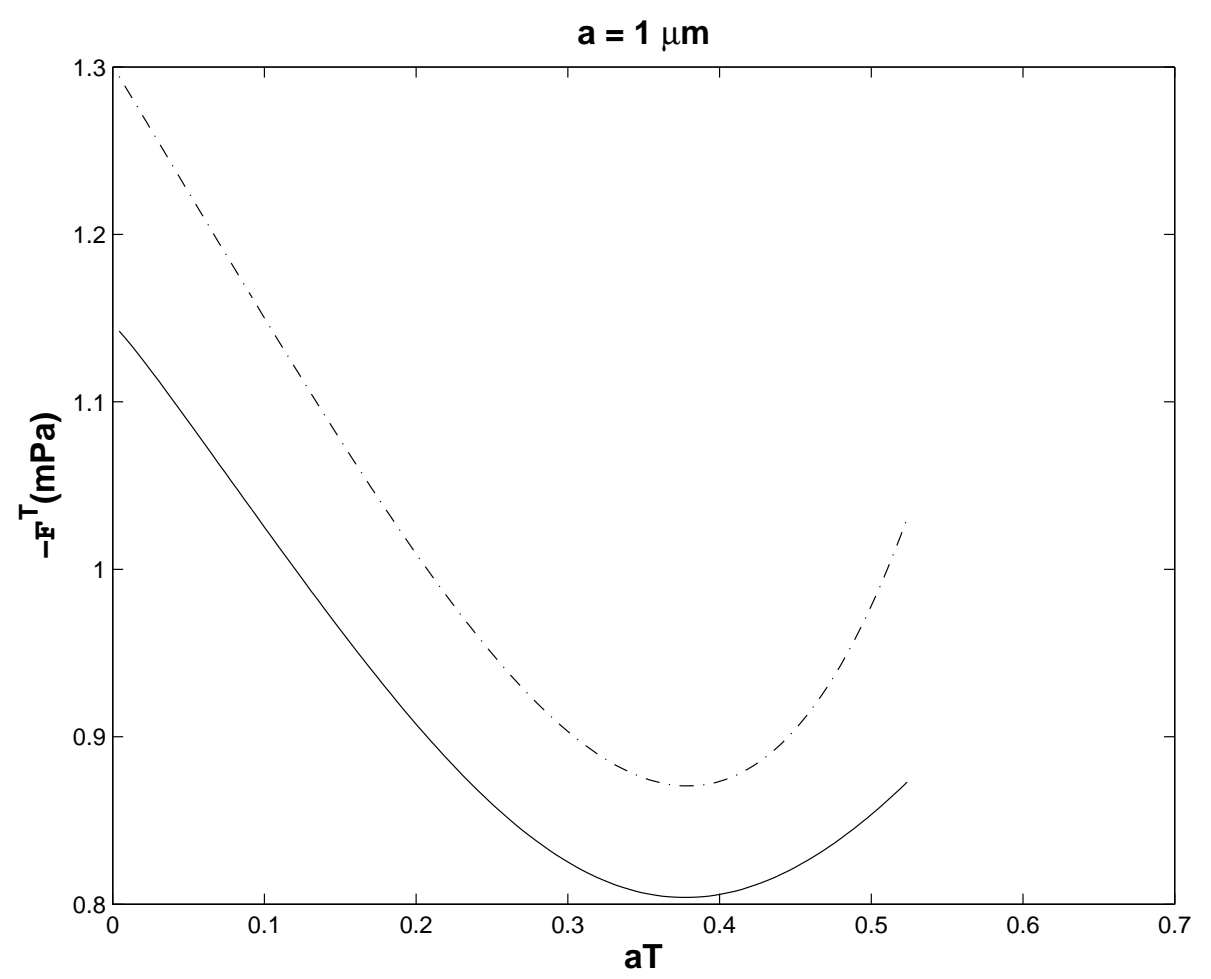

FIG. 2: Magnitude of surface force density for gold, in the temperature interval $10 K \leq T \leq 1200$ $\mathrm{K}$, when $a=1 \mu \mathrm{m}$. Solid line is physical result calculated from Eq. (4.18) where the roomtemperature data for $\varepsilon(i \zeta)$ shown in Fig. 1 are used. Broken line is calculated from the ideal low-temperature form (2.10).

corresponds to $T=300 \mathrm{~K}(\gamma=3.29, N=4)$. The deviations between the full dispersive result and Eq. (2.10) are now smaller than previously, about 5\%.

As experiments are usually made at room temperature for various gap widths, we show in Fig. 4how the surface force density for gold varies with $a$, at $T=300 \mathrm{~K}$. We have here chosen to multiply the ordinate with $a^{4}$. The linear slope seen for $a \geq 4 \mu \mathrm{m}$ is nearly that predicted in Eq. (4.14), which gives a slope of $2.0 \times 10^{-28} \mathrm{Nm}^{2} / \mu \mathrm{m}$. The linear region between 1 and $2 \mu \mathrm{m}$ corresponds to that in Eq. (2.10) or (4.14) (intermediate temperatures). Also shown is the prediction of the temperature dependent Drude model (Appendix (D)), when $T=300$ $\mathrm{K}$. The differences are seen to be very small. Since the Drude values for the permittivity are lower than the empirical ones at high frequencies, as seen in Fig. 1, we expect the predicted Drude forces to be slightly weaker than those based upon the empirical permittivities. This expectation is borne out in Fig. 4, the differences being large enough to be slightly visible at short distances, as we would expect since the plasma nature of the material becomes more pronounced for small distances. Note that the temperature dependence of the permittivity is irrelevant here because the temperature is fixed, unlike in Figs. 2 and 3 .

It is of interest to check the magnitude of the dispersive effect in these cases. We have therefore made a separate calculation of the expression (4.18) when $\varepsilon$ is taken to be constant. Figure 5 shows how the force varies with $a T$ in cases when $\varepsilon \in\{100,1000,10000, \infty\}$ are inserted in the expressions for $A_{m}$ and $B_{m}$ in Eq. (3.2a). Note that the $\varepsilon=\infty$ curve is obtained easily via the analytic result (3.13), with $A_{m}=B_{m}=1$ for all $m \geq 1$. With 


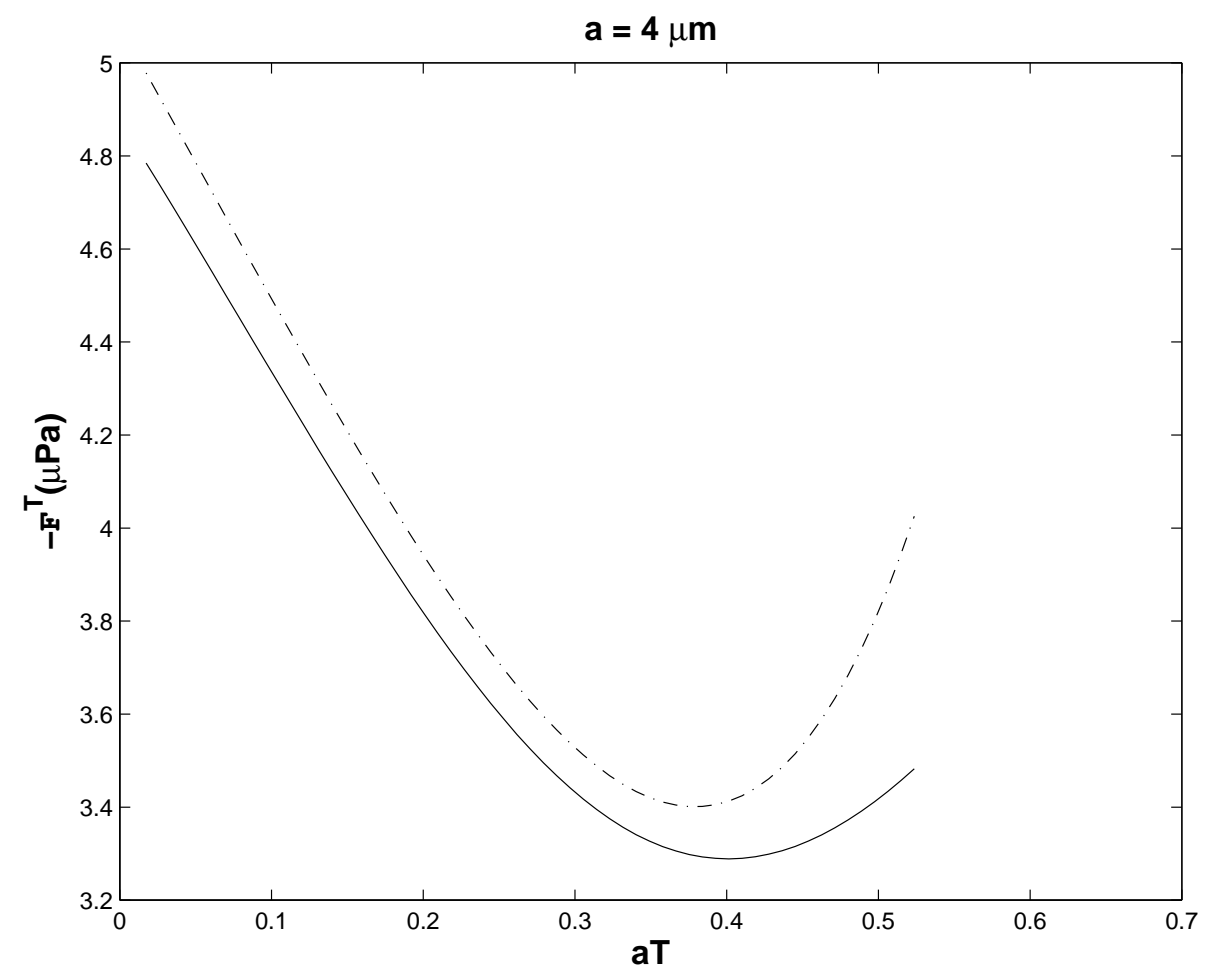

FIG. 3: Same as Fig. 2, but at a larger spacing, $a=4 \mu \mathrm{m}$, corresponding to $10 K \leq T \leq 300 \mathrm{~K}$.

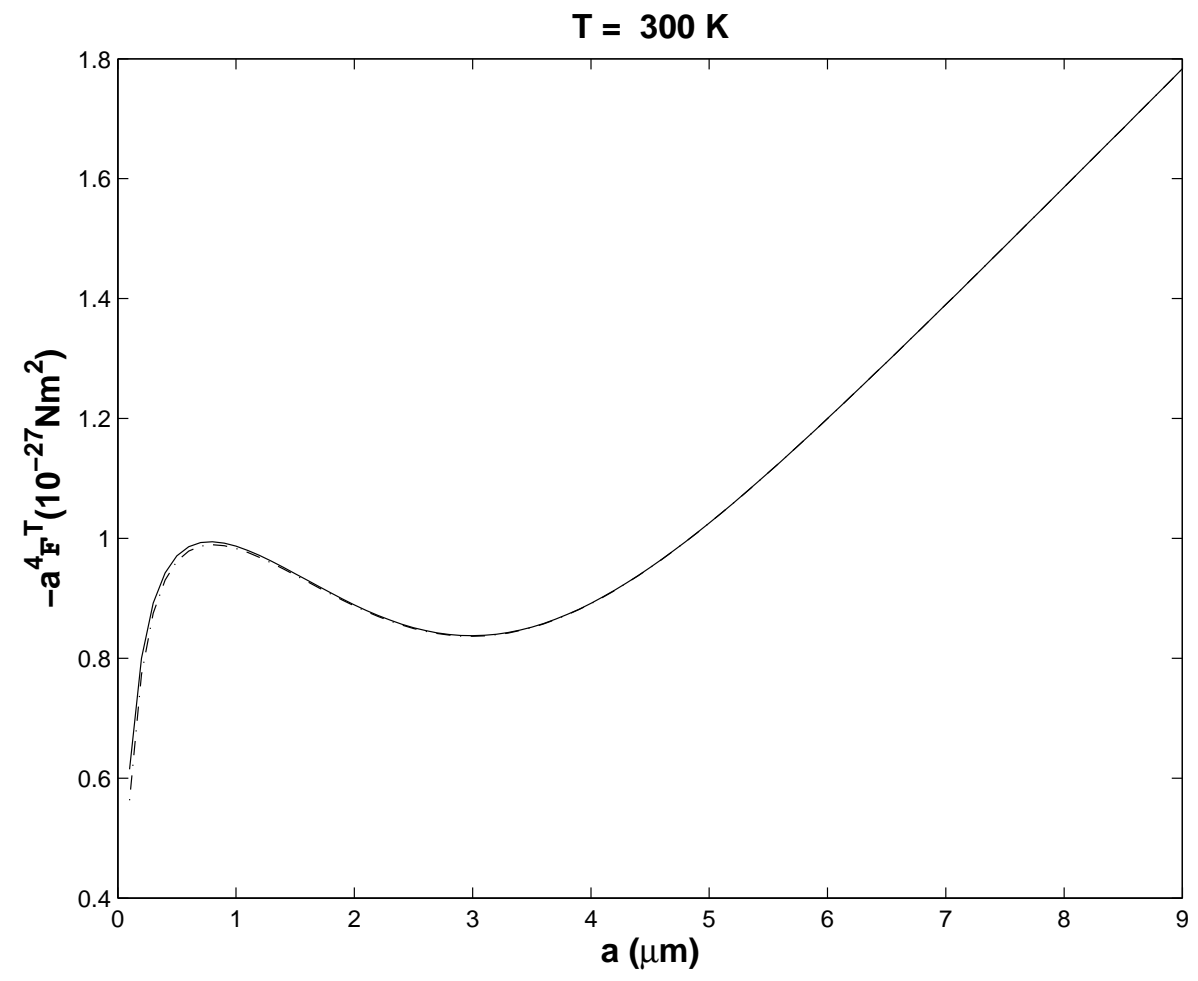

FIG. 4: Surface force density for gold, multiplied with $a^{4}$, versus $a$ when $T=300 \mathrm{~K}$. Input data for $\varepsilon(i \zeta)$ are taken from Fig. 1, 


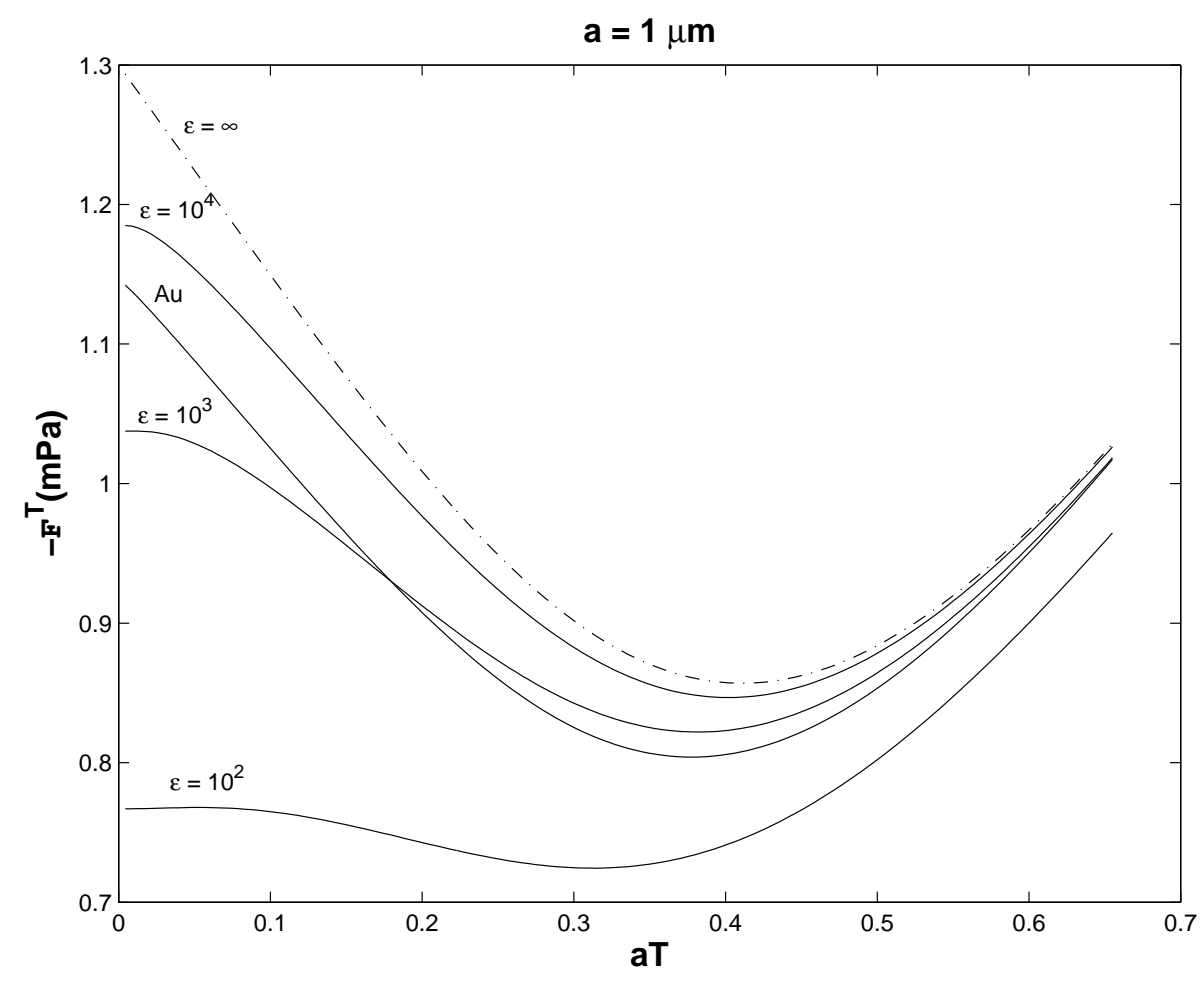

FIG. 5: Nondispersive theory: Surface force density calculated from Eq. (4.18) for $\varepsilon \in$ $\{100,1000,10000, \infty\}$. The $\varepsilon=\infty$ result is calculated from Eq. (4.22). For low values of $a T$ the latter coincides with the expression (2.10) used in Fig. 2. Also shown for comparison is the dispersive result for gold, where experimental input data for $\varepsilon(i \zeta)$ are taken from Fig. 11 Gap width is $a=1 \mu \mathrm{m}$. The constraint $a=1 \mu \mathrm{m}$ applies only to the dispersive case, since otherwise $a^{4} \mathcal{F}^{T}$ is a function of $a T$ only.

$B_{0}=0, \mathrm{Eq} \cdot(3.13)$ is modified into

$$
\mathcal{F}^{T}(\varepsilon=\infty)=\frac{1}{8 \pi \beta a^{3}}\left\{\zeta(3)-\sum_{k=1}^{\infty} \frac{1}{k^{3}}\left[s_{2}(\gamma k)+2 s_{1}(\gamma k)+2 s_{0}(\gamma k)\right]\right\},
$$

which amounts to adding the last term of Eq. (2.10). [The sum is alternatively given in Eq. (3.39), and the low-temperature limit is given in Eq. (2.10).] It is seen from the figure that the three first curves asymptotically approach the $\varepsilon=\infty$ curve, given by Eq. (4.22) when $\varepsilon$ increases, as we would expect. Again, we emphasize that the dispersive curve for gold is calculated using the available room-temperature data for $\varepsilon(i \zeta)$ from Fig. 11. In the nondispersive case, there is of course no permittivity temperature problem since $\varepsilon$ is taken to be the same for all $T$.

There are several points worth noticing from Fig. 5. (i) The curves have a horizontal slope at $T=0$. For finite $\varepsilon$ this property is clearly visible on the curves. This has to be so on physical grounds: If the force had a linear dependence on $T$ for small $T$ so would the free energy $F$, in contradiction with the requirement that the entropy $S=-\partial F / \partial T$ has to go to zero as $T \rightarrow 0$. For the gold data the initial horizontal slope is not resolvable on the scale of this graph, see the discussion at the end of Sec. IVB. (ii) The curves show that the magnitude of the force diminishes with increasing $T$ (for a fixed $a$ ), in a certain 


\begin{tabular}{|lcccccc|}
\hline \multicolumn{1}{l}{} & \multicolumn{4}{c}{$y=1$} & \multicolumn{2}{c}{$y=3$} \\
\hline$m \varepsilon\left(i \zeta_{m}\right) \times 10^{3}$ & $\zeta_{m} \times 10^{12} \mathrm{rad} / \mathrm{s}$ & $A_{m}$ & $B_{m}$ & $A_{m}$ & $B_{m}$ \\
\hline 1 & 382.0 & 8.226 & 0.9998 & 0.7899 & 0.9999 & 0.4944 \\
3 & 100.4 & 24.68 & 0.9990 & 0.8578 & 0.9997 & 0.6317 \\
5 & 49.76 & 41.13 & 0.9975 & 0.8774 & 0.9992 & 0.6759 \\
7 & 30.28 & 57.58 & 0.9956 & 0.8872 & 0.9985 & 0.6985 \\
9 & 20.52 & 74.03 & 0.9931 & 0.8930 & 0.9977 & 0.7124 \\
11 & 14.87 & 90.49 & 0.9902 & 0.8970 & 0.9967 & 0.7219 \\
13 & 11.30 & 106.9 & 0.9867 & 0.8998 & 0.9955 & 0.7288 \\
15 & 8.891 & 123.4 & 0.9827 & 0.9020 & 0.9942 & 0.7341 \\
\hline \hline
\end{tabular}

TABLE I: Some data in the dispersive theory for gold. Here $T=10 \mathrm{~K}, y \equiv q a \in\{1,3\}$. Room temperature input data for $\varepsilon(i \zeta)$ are taken from Fig. 1.

\begin{tabular}{|cccccccccc|}
\hline \hline$a(\mu \mathrm{m})$ & $m=0$ & $m=1$ & $m=2$ & $m=3$ & $m=4$ & $m=5$ & $m=6$ & $m=7$ \\
\hline 0.5 & 0.32 & 0.98 & 1.03 & 1.05 & 1.06 & 1.07 & 1.08 & 1.08 \\
1 & 0.58 & 1.98 & 2.05 & 2.07 & 2.08 & 2.08 & 2.07 & 2.06 \\
2 & 1.10 & 4.04 & 4.09 & 4.07 & 4.02 & 3.96 & 3.88 & 3.79 \\
3 & 1.63 & 6.11 & 6.09 & 5.98 & 5.80 & 5.59 & 5.36 & 5.10 \\
4 & 2.16 & 8.18 & 8.04 & 7.75 & 7.37 & 6.93 & 6.45 & 5.95 \\
5 & 2.69 & 10.24 & 9.92 & 9.37 & 8.69 & 7.94 & 7.16 & 6.38 \\
6 & 3.23 & 12.30 & 11.71 & 10.81 & 9.75 & 8.63 & 7.51 & 6.45 \\
7 & 3.78 & 14.33 & 13.39 & 12.06 & 10.55 & 9.02 & 7.56 & 6.24 \\
\hline \hline
\end{tabular}

TABLE II: Contribution from the various Matsubara frequencies for gold. What is given is the percentage of $\mathcal{F}^{T}$ for each mode in the region $m \in[0,7]$. The temperature is $T=10 \mathrm{~K}$. Room temperature input data for $\varepsilon(i \zeta)$ are taken from Fig. 1.

temperature interval up to $a T \simeq 0.3$. This perhaps counterintuitive effect is thus clear from the nondispersive curves as well as from the dispersive curves in Figs. 2 and 3 , (iii) It is seen that the curve for $\varepsilon=$ const. $=1000$ gives a reasonably good approximation to the real dispersive curve for gold when $a=1 \mu \mathrm{m}$; the deviations are less than about $5 \%$ except for the lowest values of $a T(a T<0.1)$. This fact makes our neglect of the temperature dependence of $\varepsilon(i \zeta)$ appear physically reasonable; the various curves turn out to be rather insensitive with respect to variations in the input values of $\varepsilon(i \zeta)$. (iv) One notes that the curves (for large $\varepsilon$ ) in Fig. 5 are consistent with the free energy (4.14) using the rough approximation (4.9) for $B_{m}$. Especially one notes the initial decrease of the magnitude of the Casimir force for increasing $T$ when $\varepsilon$ is large. As discussed below Eq. (4.12) this is again connected with the counterintuitive negative contribution to the entropy. (v) Also, it can be remarked that $B_{0}=0$ is required when $\varepsilon$ is finite. Otherwise the curves in Fig. 5, and thus the free energy, would have a finite slope at $T=0$ which again would imply a finite entropy contribution at $T=0$ in violation with the third law of thermodynamics.

Instead of confining ourselves to a "black box" calculation of the force expression (4.18), it is desirable to break up the expression somewhat, to see how the various values of $m$ contribute. We do this in Tables 【III, for gold. The first two tables refer to the case $T=10$ 


\begin{tabular}{|ccccccccc|}
\hline \hline$a(\mu \mathrm{m})$ & $m=0$ & $m=1$ & $m=2$ & $m=3$ & $m=4$ & $m=5$ & $m=6$ & $m=7$ \\
\hline 0.5 & 10.20 & 31.24 & 22.95 & 15.09 & 9.18 & 5.28 & 2.91 & 1.55 \\
1 & 20.07 & 49.37 & 20.83 & 6.97 & 2.03 & 0.54 & 0.14 & 0.03 \\
2 & 44.56 & 49.87 & 5.17 & 0.37 & 0.02 & & & \\
3 & 70.95 & 28.41 & 0.63 & 0.01 & & & & \\
4 & 88.88 & 11.07 & 0.05 & & & & & \\
5 & 96.58 & 3.42 & & & & & & \\
6 & 99.06 & 0.94 & & & & & \\
7 & 99.76 & 0.24 & & & & & \\
\hline \hline
\end{tabular}

TABLE III: Same as in Table II but at temperature 300 K. Data from Fig. 1 is again used.

K. (Again, the experimental values of $\varepsilon(i \zeta)$ at room temperature are used.) As $y$ is the important integration parameter in Eq. (4.18), we keep $y$ fixed in Table $\mathbb{1}, y \in\{1,3\}$. It is seen that $A_{m}$ stays close to 1 , whereas $B_{m}$ decreases for increasing $y$, if $m$ is kept constant. Table shows how the various $m$ contribute to the force. Writing the total force as a sum,

$$
\mathcal{F}^{T}=\sum_{m=0}^{\infty} \mathcal{F}_{m}^{T},
$$

the columns in the table show the percentage of $\mathcal{F}_{m}^{T}$, i.e., $\left(\mathcal{F}_{m}^{T} / \mathcal{F}^{T}\right) \times 100$, distributed over the region $m \in[0,7]$, when $T=10 \mathrm{~K}$. The distribution from the various $m \mathrm{~s}$ is seen to be very broad, as is characteristic for a low-temperature problem. Table III shows the same kind of distribution over $m$ when $T=300 \mathrm{~K}$. Already from a gap distance of $a=3-4 \mu \mathrm{m}$ onwards, the distribution is heavily concentrated around low $m$, as is characteristic of a high-temperature problem.

It is in this context instructive as a corollary to go back to the integral over $y$ in Eq. (4.18). One would expext the main contribution to the integral to come from the region $y=q a=$ $\sqrt{k_{\perp}^{2}+\zeta^{2}} a \sim 1$. Assuming the most important values of $k_{\perp}$ to be moderate, this means $\zeta a \sim 1$, or $m \sim 1 /(2 \pi a T)$, since $\zeta=2 \pi m T$. When $T=300 \mathrm{~K}$, we thus expect the dominant contribution to come from $m \sim 1$ when $a=1 \mu \mathrm{m}$, and from $m=0$ when $a \geq 3 \mu \mathrm{m}$. This is seen to agree very well with the data in Table III. Similar considerations apply to the case $T=10 \mathrm{~K}$, although the contributions from the various $m$ s are then more smeared out.

The important question is now: Have the characteristic temperature variations shown in the theoretical figures above been verified in practice? Of most interest in this context is the experiment of Bressi et al. [26], since it deals with parallel plates directly. According to personal information from R. Onofrio, one of the members of the Italian group, the observed Casimir forces were lower than those predicted by the traditional (SDM) theory for conducting plates, in cases where the distances were low, $a \leq 0.5 \mu \mathrm{m}$. This reduction effect is apparent also from their Fig. 4. Now, the plates in this experiment were coated with chromium rather than with gold, but we can check that the corrections in that case are of the same magnitude as if the plates were coated with gold. Namely, an explicit calculation of the analogue of Fig. 5 for the case $a=0.5 \mu \mathrm{m}$ (not shown here) shows that at room temperature for which $a T=0.065$, the force becomes $-\mathcal{F}^{T}=15.5 \mathrm{mPa}$. The conventional $(\mathrm{SDM})$ theory gives in this case the force $1.3 \times 2^{4}=20.8 \mathrm{mPa}$. The predicted reduction in the force is thus about $25 \%$, somewhat more than the measurements indicate. In any case, 
this suggests that the reduced force seen at room temperature in Ref. 26] may be the first actual observation of the temperature effect predicted theoretically.

At larger distances, however, between 1 and $2 \mu \mathrm{m}$, the situation is no longer so clear-cut, since they observe a Casimir force in excess of the theoretically predicted one. The reason for this deviation is not known. Of course the force becomes weaker at larger distances, thus being subject to larger experimental uncertainties. The most natural conclusion to be drawn at this stage is that we have to wait for better precision in this kind of difficult experiment. Ideas for such an improved experiment which could descriminate between the different models have just appeared [46].

\section{APPENDIX A: ON THE SMOOTHNESS OF THE REFLECTION COEFFI- CIENT $r_{2}$ AT LOW FREQUENCIES, FOR A METAL}

In view of the current discussion in the literature about the value of the reflection coefficient $r_{2}$ for a metal in the limit of low frequencies, let us consider this point in some detail. As mentioned earlier, the problem occurs in connection with use of the Drude formula, Eq. (1.1). The coefficient $r_{2}$ is actually the square root of our quantity $B_{m}$ defined in Eq. (3.2a), so that we may write

$$
r_{2}^{2}=\left(\frac{s-p}{s+p}\right)^{2}
$$

Let us keep the transverse wave vector $\mathbf{k}_{\perp}$ fixed, and perform a power series expansion of $\varepsilon(i \zeta)$ to the first order in $\zeta / \nu$. [Any normal metal must have a finite relaxation frequency $\nu$, so that in the limit of low frequencies, $\zeta / \nu$ can be regarded as small. At zero temperature, we are assuming $\nu(T=0) \neq 0$.] From Eq. (1.1) we get

$$
\varepsilon(i \zeta)-1 \rightarrow \frac{\omega_{p}^{2}}{\nu \zeta}\left(1-\frac{\zeta}{\nu}\right),
$$

which for the Lifshitz variables $s$ and $p$ implies [cf. Eq. (3.2b)]

$$
\begin{aligned}
& s=\sqrt{\varepsilon-1+p^{2}} \rightarrow \frac{k_{\perp}}{\zeta}\left(1+\frac{\omega_{p}^{2} \zeta}{2 \nu k_{\perp}^{2}}\right), \\
& p=\frac{k_{\perp}}{\zeta} \sqrt{1+\frac{\zeta^{2}}{k_{\perp}^{2}}} \rightarrow \frac{k_{\perp}}{\zeta} .
\end{aligned}
$$

Insertion into Eq. (A1) now yields

$$
r_{2}^{2} \rightarrow\left(\frac{\omega_{p}^{2}}{4 k_{\perp}^{2}}\right)^{2}\left(\frac{\zeta}{\nu}\right)^{2} .
$$

We thus see that $r_{2}^{2} \rightarrow 0$ smoothly as $\zeta \rightarrow 0$. Contrary to recent statements in the literature 6, 7, 8], we find that there is no peculiar effect taking place at $\zeta=0$, when the Drude model is used. The result (A4) corresponds to a vanishing contribution to the Casimir effect from the $m=0$ TE mode for a real metal, in accordance with our treatment in Sec. IV.

The argument above hinged on the assumption that $\mathbf{k}_{\perp} \neq \mathbf{0}$. One might wonder: What happens if $\mathbf{k}_{\perp}$ is exactly zero? Mathematically, it then follows from Eq. (3.2b) that $r_{2}^{2}=1$. This case cannot, however, be of physical importance. The set $\mathbf{k}_{\perp}=\mathbf{0}$ is mathematically of measure zero, and has thus no influence upon real physics. 


\section{APPENDIX B: ON THE PHYSICAL IMPORTANCE OF $A_{m}$ AND $B_{m}$}

It is physically instructive to show in some detail how the coefficients $A_{m}$ and $B_{m}$ relate to the conventional Fresnel coefficients in optics, at oblique incidence. Consider first the TM mode, and let a plane wave be incident from the left (medium 1 , refractive index $n_{1}=\sqrt{\varepsilon}$ ) at a real angle of incidence $\theta_{i}$ towards the boundary located at $z=0$. The angle of transmission to the vacuum region $z>0$ is $\theta_{t}$. For instance from Ref. [47] we have for the ratio between the reflected wave amplitude $R^{\mathrm{TM}}$ and the incident wave amplitude $A^{\mathrm{TM}}$

$$
\frac{R^{\mathrm{TM}}}{A^{\mathrm{TM}}}=\frac{\cos \theta_{i}-n_{1} \cos \theta_{t}}{\cos \theta_{i}+n_{1} \cos \theta_{t}}
$$

Since $\cos \theta_{i}=\sqrt{1-k_{\perp}^{2} /\left(\varepsilon \omega^{2}\right)}, \cos \theta_{t}=\sqrt{1-k_{\perp}^{2} / \omega^{2}}$ we get, when replacing $\omega$ by $i \zeta$,

$$
\frac{R^{\mathrm{TM}}}{A^{\mathrm{TM}}}=\frac{\sqrt{\varepsilon+k_{\perp}^{2} / \zeta^{2}}-\varepsilon \sqrt{1+k_{\perp}^{2} / \zeta^{2}}}{\sqrt{\varepsilon+k_{\perp}^{2} / \zeta^{2}}+\varepsilon \sqrt{1+k_{\perp}^{2} / \zeta^{2}}} .
$$

Now $s=\sqrt{\varepsilon-1+p^{2}}=\sqrt{\varepsilon+k_{\perp}^{2} / \zeta^{2}}, p=q / \zeta=\sqrt{1+k_{\perp}^{2} / \zeta^{2}}$, and so we get

$$
\frac{R^{\mathrm{TM}}}{A^{\mathrm{TM}}}=\frac{s-\varepsilon p}{s+\varepsilon p}=\sqrt{A_{m}}
$$

Similarly for the TE mode,

$$
\frac{R^{\mathrm{TE}}}{A^{\mathrm{TE}}}=\frac{s-p}{s+p}=\sqrt{B_{m}}
$$

Of course, these results are also found in textbooks [48].

\section{APPENDIX C: PARALLEL DIELECTRICS}

In Ref. [1] the following result for the TE reduced Green's function is given,

$$
g^{H}\left(z, z^{\prime}\right)=\frac{1}{2 \kappa_{2}}\left(e^{-\kappa_{2}\left|z-z^{\prime}\right|}+r e^{-\kappa_{2}\left(z+z^{\prime}-2 a\right)}\right) .
$$

which is valid for $z, z^{\prime}>a$. Here the reflection coefficient is

$$
\begin{aligned}
& r=\frac{\kappa_{2}-\kappa_{3}}{\kappa_{2}+\kappa_{3}}+\frac{4 \kappa_{2} \kappa_{3}}{\kappa_{3}^{2}-\kappa_{2}^{2}} d^{-1}, \\
& d=\frac{\kappa_{3}+\kappa_{1}}{\kappa_{3}-\kappa_{1}} \frac{\kappa_{3}+\kappa_{2}}{\kappa_{3}-\kappa_{2}} e^{2 \kappa_{3} a}-1,
\end{aligned}
$$

and

$$
\kappa_{i}^{2}=k^{2}-\omega^{2} \epsilon_{i}
$$

and we have taken a parallel dielectric slab geometry

$$
\epsilon(z)=\left\{\begin{array}{l}
\epsilon_{1}, \quad z<0 \\
\epsilon_{3}, \quad 0<z<a \\
\epsilon_{2}, \quad a<z
\end{array}\right.
$$


The temperature controversy centers on the zero mode. If $\omega^{2} \epsilon$ vanishes at $\omega=0$ (true for the Drude model, but not the plasma model), then the reflection coefficient vanishes there, $r=0$, and we have only a free Green's function at $\omega=0$, that is, the boundary becomes transparent. The TM reflection coefficient does not have this property.

We have redone the calculation to find the reduced Green's function in the interior region, $0<z, z^{\prime}<a$. We find

$$
\begin{aligned}
g^{H}\left(z, z^{\prime}\right)=\frac{1}{2 \kappa_{3}}\left\{e^{-\kappa_{3}\left|z-z^{\prime}\right|}+\frac{\kappa_{3}-\kappa_{1}}{\kappa_{3}+\kappa_{1}} e^{-\kappa_{3}\left(z+z^{\prime}\right)}+d^{-1}\left[e^{\kappa_{3}\left(z-z^{\prime}\right)}+e^{\kappa_{3}\left(z^{\prime}-z\right)}\right.\right. \\
\left.\left.+\frac{\kappa_{3}+\kappa_{1}}{\kappa_{3}-\kappa_{1}} e^{\kappa_{3}\left(z+z^{\prime}\right)}+\frac{\kappa_{3}-\kappa_{1}}{\kappa_{3}+\kappa_{1}} e^{-\kappa_{3}\left(z+z^{\prime}\right)}\right]\right\} .
\end{aligned}
$$

Again, it is easy to see that we obtain only the free Green's function for the zero mode:

$$
g^{H}\left(z, z^{\prime} ; \omega=0\right)=\frac{1}{2 k} e^{-k\left|z-z^{\prime}\right|},
$$

provided $\lim _{\omega \rightarrow 0} \omega^{2} \epsilon(\omega)=0$.

A check of this result is that if we substitute Eq. (C5) into the expression for the force/area (3.13) of Ref. 1], we get for the TE contribution to the force [see Eq. (3.10) there]

$$
\begin{aligned}
\mathcal{F}^{T} & =\frac{i}{2} \int \frac{d \omega}{2 \pi} \frac{(d \mathbf{k})}{(2 \pi)^{3}}\left(\epsilon_{2}-\epsilon_{3}\right) \omega^{2} g^{H}(a, a) \\
& =\frac{i}{2} \int \frac{d \omega}{2 \pi} \frac{(d \mathbf{k})}{(2 \pi)^{3}}\left(\kappa_{3}-\kappa_{2}+2 \kappa_{3} d^{-1}\right),
\end{aligned}
$$

identical to the first term in Eq. (3.19) of Ref. [1], and apart from a contact term is the same as the second term in Eq. (3.1). All of this does not seem to support the claims of Klimchitskaya et al. [6, 7, 8, 38] that there is something ill-defined about the $\omega=0$ limit.

\section{APPENDIX D: TEMPERATURE DEPENDENCE OF THE RELAXATION FRE- QUENCY FOR GOLD}

To investigate the temperature dependence of the relaxation frequency $\nu(T)$ in the Drude relation

$$
\varepsilon(i \zeta, T)=1+\frac{\omega_{p}^{2}}{\zeta[\zeta+\nu(T)]}
$$

for gold, it is convenient to make use of the Bloch-Grüneisen formula for the temperature dependence of the electrical resistivity $\rho$ [49]:

$$
\rho(T)=C\left(\frac{T}{\Theta}\right)^{5} \int_{0}^{\Theta / T} \frac{x^{5} e^{x} d x}{\left(e^{x}-1\right)^{2}} .
$$

It is known that $\Theta=175 \mathrm{~K}$ for gold. The constant $C$ can be determined from the knowledge that $\rho=2.20 \times 10^{-8} \Omega \mathrm{m}$ at temperature $295 \mathrm{~K}[50]$. We obtain $C=5.32 \times 10^{-8} \Omega \mathrm{m}$.

The theoretical relationship between $\nu$ and the static resistivity $\rho$ is

$$
\nu=\frac{f_{0} N_{e} e^{2}}{m} \rho,
$$




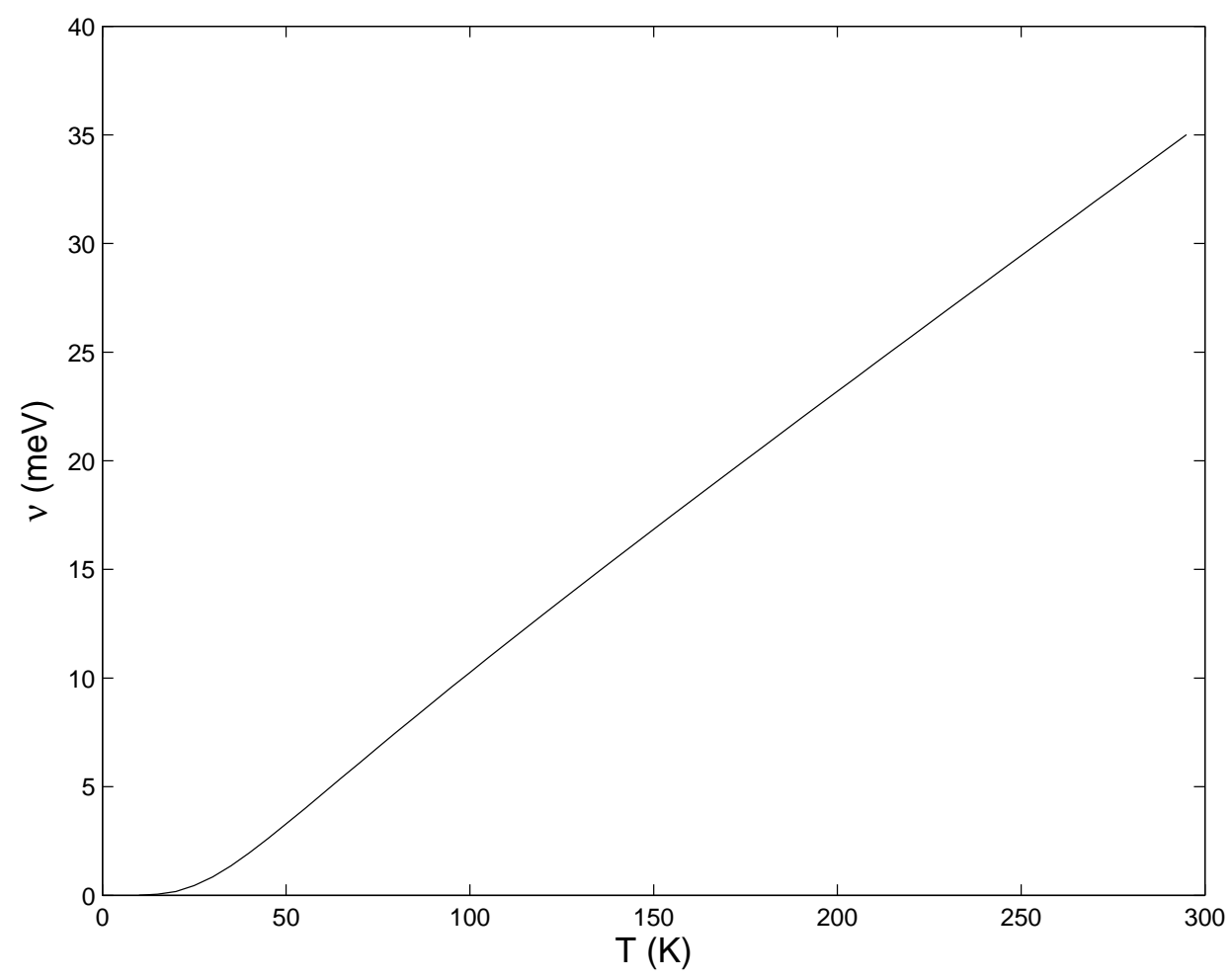

FIG. 6: Temperature dependence of the relaxation frequency for gold.

where $N_{e}$ is the number density of atoms, $f_{0} N_{e}$ with $f_{0} \sim 1$ the number density of free electrons, and $m$ the effective electron mass. The simplest way to proceed is to put $\nu=K \rho$ with $K$ a constant, and make use of the room-temperature data of Eq. (4.16). We obtain

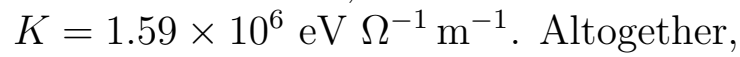

$$
\nu(T)=0.0847\left(\frac{T}{\Theta}\right)^{5} \int_{0}^{\Theta / T} \frac{x^{5} e^{x} d x}{\left(e^{x}-1\right)^{2}},
$$

where the unit of $\nu(T)$ is $\mathrm{eV}$. The temperature variation is shown in Fig. 6. For low temperatures, $\nu(T) \propto T^{5}$, whereas at high temperatures, $\nu(T) \propto T$. The curve is seen to be similar to the one given in Fig. 3 of Ref. [9], in the case of aluminum.

An important caveat must be mentioned, however; these formulas neglect the effect of impurities, which give rise to a nonzero resistivity at zero temperature [51]. This makes the use of these ideal resistivity models questionable, and adds further evidence that the behavior of the entropy discussed in Sec. IV is correct.

\section{ACKNOWLEDGMENTS}

KAM is grateful to the US Department of Energy for partial financial support of this research. He would like to thank Peter van Nieuwenhuizen for discussion about the subtleties of zero modes. IB thanks Astrid Lambrecht and Serge Reynaud for providing their numerical calculations of the dispersion relation for gold, Roberto Onofrio for information about the experiment of Ref. [26], Vladimir Mostepanenko for valuable discussions on the relaxation 
frequency temperature problem, as well as for information about numerical data, and Bo Sernelius for discussions about the resistivity of metals at very low temperatures.

[1] K. A. Milton, The Casimir Effect: Physical Manifestations of the Zero-Point Energy (World Scientific, Singapore, 2001).

[2] V. M. Mostepanenko and N. N. Trunov, The Casimir Effect and its Applications (Clarendon Press, Oxford, 1997).

[3] P. W. Milonni, The Quantum Vacuum (Academic Press, San Diego, 1994).

[4] G. Plunien, B. Müller, and W. Greiner, Phys. Reports 134, 87 (1986).

[5] M. Bordag, U. Mohideen, and V. M. Mostepanenko, Phys. Reports 353, 1 (2001).

[6] G. L. Klimchitskaya and V. M. Mostepanenko, Phys. Rev. A 63, 062108 (2001).

[7] M. Bordag , B. Geyer, G. L. Klimchitskaya, and V. M. Mostepanenko, Phys. Rev. Lett. 85, $503(2000)$.

[8] E. Fischbach, D. E. Krause, V. M. Mostepanenko, and M. Novello, Phys. Rev. D 64, 075010 (2001).

[9] V. B. Bezerra, G. L. Klimchitskaya, and V. M. Mostepanenko, Phys. Rev. A 66, 062112 (2002).

[10] E. M. Lifshitz and L. P. Pitaevskii, Statistical Physics, Part 2 (Pergamon Press, Oxford, 1980), Sec. 81.

[11] J. Schwinger, L. L. DeRaad, Jr., and K. A. Milton, Ann. Phys. (N.Y.) 115, 1 (1978).

[12] J. S. Høye, I. Brevik, and J. B. Aarseth, Phys. Rev. E 63, 051101 (2001).

[13] M. Boström and Bo E. Sernelius, Phys. Rev. Lett. 84, 4757 (2000).

[14] I. Brevik, J. B. Aarseth, and J. S. Høye, Int. J. Mod. Phys. A 17, 776 (2002).

[15] I. Brevik, J. B. Aarseth, and J. S. Høye, Phys. Rev. E 66, 026119 (2002).

[16] S. K. Lamoreaux, quant-ph/0007029 v4.

[17] Bo E. Sernelius and M. Boström, Phys. Rev. Lett. 87, 259101 (2001).

[18] M. Bordag, B. Geyer, G. L. Klimchitskaya, and V. M. Mostepanenko, Phys. Rev. Lett. 87, 259102 (2001).

[19] S. K. Lamoreaux, Phys. Rev. Lett. 78, 5 (1997).

[20] U. Mohideen and A. Roy, Phys. Rev. Lett. 81, 4549 (1998).

[21] A. Roy, C.-Y. Lin, and U. Mohideen, Phys. Rev. D 60, R111101 (1999).

[22] B. W. Harris, F. Chen, and U. Mohideen, Phys. Rev. A 62, 052109 (2000).

[23] F. Chen, U. Mohideen, G. L. Klimchitskaya, and V. M. Mostepanenko, Phys. Rev. Lett. 88, 101801 (2002).

[24] T. Ederth, Phys. Rev. A 62, 062104 (2000).

[25] H. B. Chan, V. A. Aksyuk, R. N. Kleiman, D. J. Bishop, and F. Capasso, Phys. Rev. Lett. 87, 211801 (2001); Science 291, 1941 (2001).

[26] G. Bressi, G. Carugno, R. Onofrio, and G. Ruoso, Phys. Rev. Lett. 88, 041804 (2002).

[27] A. Lambrecht and S. Reynaud, in Seminaire Poincare 1 (Institut Henri Poincare, Paris, 9 March 2002), pp. 79-92 [www.lpthe.jussieu.fr/poincare/], quant-ph/0302073.

[28] J. Blocki, J. Randrup, W. J. Swialecki, and C. F. Tsang, Ann. Phys. (N.Y.) 105, 427 (1977).

[29] S. K. Lamoreaux, Phys. Rev. Lett. 81, 5475 (1998); Phys. Rev. A 59, R3149 (1999).

[30] A. Lambrecht and S. Reynaud, Eur. Phys. J. D 8, 309 (2000); Phys. Rev. Lett. 84, 5672 (2000). 
[31] C. Genet, A. Lambrecht, and S. Reynaud, Int. J. Mod. Phys. A 17, 761 (2002).

[32] V. B. Svetovoy and M. V. Lokhanin, Mod. Phys. Lett. A 15, 1437 (2000); quant-ph/0004004 Phys. Lett. A 280, 177 (2001).

[33] G. Barton, Phys. Rev. A 64, 032103 (2001).

[34] J. Feinberg, A. Mann, and M. Revzen, Ann. Phys. (N.Y.) 288, 103 (2001).

[35] V. B. Bezerra, G. L. Klimchitskaya, and V. M. Mostepanenko, Phys. Rev. A 65, 052113 (2002).

[36] J. R. Torgerson and S. K. Lamoreaux, quant-ph/0208042

[37] P. C. Martin and J. Schwinger, Phys. Rev. 115, 1342 (1959).

[38] G. L. Klimchitskaya, Int. J. Mod. Phys. A 17, 751 (2002).

[39] J. S. Høye and I. Brevik, Physica (Amsterdam) A 259, 165 (1998).

[40] F. Sauer, PhD thesis, Göttingen, 1962.

[41] J. Mehra, Physica (Amsterdam) 37, 145 (1967).

[42] J. S. Høye and G. Stell, J. Chem. Phys. 75, 5133 (1981).

[43] I. Brevik and J. S. Høye, Physica A 153, 420 (1988).

[44] I. Brevik, Phys. Reports 52, 133 (1979).

[45] Handbook of Optical Constants of Solids, edited by E. D. Palik (Academic Press, New York, 1998).

[46] F. Chen, G. L. Klimchitskaya, U. Mohideen, and V. M. Mostepanenko, quant-ph/0302149, to appear in Phys. Rev. Lett.

[47] M. Born and E. Wolf, Principles of Optics, 6th ed. (Pergamon Press, Oxford, 1980), p. 40.

[48] J. Schwinger, L. L. DeRaad, Jr., K. A. Milton, and W.-y. Tsai, Classical Electrodynamics (Perseus, New York, 1998).

[49] See, for instance, Handbook of Physics, edited by E. U. Condon and H. Odishaw, 2nd ed. (McGraw-Hill, New York, 1967), Eq. (6.12).

[50] American Institute of Physics Handbook, edited by D. E. Gray, 3rd ed. (McGraw-Hill, New York, 1972).

[51] M. Khoshenevisan, W. P. Pratt, Jr., P. A. Schroeder, and S. D. Steenwyk, Phys. Rev. B 19, 3873 (1979). 